\title{
Renormalizing vector currents in lattice QCD using momentum-subtraction schemes
}

\author{
D. Hatton $\odot,{ }^{1, *}$ C. T. H. Davies $\odot,{ }^{1, \dagger}$ G. P. Lepage, ${ }^{2}$ and A. T. Lytle $\oplus^{3}$ \\ (HPQCD Collaboration) \\ ${ }^{1}$ SUPA, School of Physics and Astronomy, University of Glasgow, Glasgow, G12 8QQ, United Kingdom \\ ${ }^{2}$ Laboratory for Elementary-Particle Physics, Cornell University, Ithaca, New York 14853, USA \\ ${ }^{3}$ INFN, Sezione di Roma Tor Vergata, Via della Ricerca Scientifica 1, 00133 Roma RM, Italy
}

(Received 9 September 2019; published 20 December 2019)

\begin{abstract}
We examine the renormalization of flavor-diagonal vector currents in lattice QCD with the aim of understanding and quantifying the systematic errors from nonperturbative artifacts associated with the use of intermediate momentum-subtraction schemes. Our study uses the highly improved staggered quark action on gluon-field configurations that include $n_{f}=2+1+1$ flavors of sea quarks, but our results have applicability to other quark actions. Renormalization schemes that make use of the exact lattice vector Ward-Takahashi identity for the conserved current also have renormalization factors, $Z_{V}$, for nonconserved vector currents that are free of contamination by nonperturbative condensates. We show this by explicit comparison of two such schemes: that of the vector form factor at zero momentum transfer and the RI-SMOM momentum-subtraction scheme. The two determinations of $Z_{V}$ differ only by discretization effects (for any value of momentum transfer in the RI-SMOM case). The RI'-MOM scheme, although widely used, does not share this property. We show that $Z_{V}$ determined in the standard way in this scheme has $\mathcal{O}(1 \%)$ nonperturbative contamination that limits its accuracy. Instead we define an $\mathrm{RI}^{\prime}-\mathrm{MOM} Z_{V}$ from a ratio of local to conserved vector current vertex functions and show that this $Z_{V}$ is a safe one to use in lattice QCD calculations. We also perform a first study of vector current renormalization with the inclusion of quenched QED effects on the lattice using the RI-SMOM scheme.
\end{abstract}

DOI: $10.1103 /$ PhysRevD.100.114513

\section{INTRODUCTION}

Lattice QCD is the method of choice for the accurate calculation of hadronic matrix elements needed for a huge range of precision particle physics phenomenology aimed at uncovering new physics. Compelling evidence of new physics in the comparison of experiment to the Standard Model has so far proved elusive, however, and this is driving the need for smaller and smaller uncertainties on both sides. This means that the error bars from lattice QCD calculations must be reduced to sub- $1 \%$ levels. Here we address uncertainties coming from the renormalization of lattice QCD operators to match their continuum QCD

\footnotetext{
*d.hatton.1@ research.gla.ac.uk †christine.davies@glasgow.ac.uk

"http://www.physics.gla.ac.uk/HPQCD.
}

Published by the American Physical Society under the terms of the Creative Commons Attribution 4.0 International license. Further distribution of this work must maintain attribution to the author(s) and the published article's title, journal citation, and DOI. Funded by SCOAP ${ }^{3}$. counterparts. This renormalization is needed so that the hadronic matrix elements of the operators calculated in lattice QCD can be used in continuum phenomenology. Ideally the uncertainty from the renormalization factors, $Z$, should be much less than other lattice QCD uncertainties (such as statistical errors) in the hadronic matrix element calculation.

Defining QCD on a space-time lattice provides an ultraviolet cutoff on the theory of $\pi / a$ where $a$ is the lattice spacing. This is a different regularization than that used in continuum formulations of QCD and hence we expect a finite renormalization to be required to match lattice QCD and continuum operators. This renormalization takes account of the differing ultraviolet behavior in the two cases and hence can be calculated as a perturbative series in the strong coupling constant, $\alpha_{s}$, at a scale related to the ultraviolet cutoff. Lattice QCD perturbation theory is notoriously difficult, however, and very few renormalization constants have been calculated beyond $\mathcal{O}\left(\alpha_{s}\right)$ (for an example of a twoloop renormalization in lattice QCD perturbation theory see Ref. [1]). It therefore seems clear that this route will not give accurate enough results for the future. 
Instead we concentrate here on other approaches that can be implemented using results from within the nonperturbative lattice QCD calculation. These approaches will typically still need to make use of perturbation theory to provide a full matching to a preferred continuum scheme such as $\overline{\mathrm{MS}}$, but if this perturbation theory can be done in the continuum to high order then much improved accuracy should be possible.

At the heart of these nonperturbative-on-the-lattice approaches is always the idea that we can construct a short-distance operator on the lattice whose leading term in an operator product expansion is the operator that we wish to study. The matrix elements that we calculate on the lattice, and use to determine $Z$, will be dominated by those from the leading operator. There will inevitably be contamination, however, from subleading terms in the expansion, i.e., higher-dimension operators multiplied by inverse powers of some scale. This means then that nonperturbative artifacts can enter the determination of $Z$ and these must be understood and controlled in order to make use of the $Z$ obtained [2].

Here we will study the renormalization factor $Z_{V}$ associated with the flavor-diagonal vector current that couples to the photon. This current is conserved in continuum QCD and has no anomalous dimension. Hence we can study the lattice QCD determination of $Z_{V}$ directly, and its dependence on the lattice spacing, without having to combine it with a matrix element for the vector current determined in lattice QCD. $Z_{V}$ is a special case of a renormalization constant that can be calculated exactly in lattice QCD, i.e., without the need for any continuum perturbation theory and without nonperturbative artifact contamination. It is important to use a method that allows for such a calculation if we want an accurate normalization.

It is possible to write down conserved vector currents in lattice QCD and use these, knowing that they do not require renormalization because there is an exact vector WardTakahashi identity. Conserved vector currents are not generally used, however, because they are complicated objects, especially for discretizations of QCD that are highly improved. The removal of tree-level discretization errors at $\mathcal{O}\left(a^{2}\right)$ from the covariant derivative in the Dirac equation requires the addition of operators that extend over three links [3]. The conserved current then contains both one-link and three-link terms and this is the case for the highly improved staggered quark (HISQ) action that we will use here (see the Appendix A). We demonstrate explicitly how the vector Ward-Takahashi identity works in this case.

The HISQ action was designed [4] to have very small discretization effects and this allows it to be used to study both light and heavy quark phenomenology [5]. Whenever a vector current is needed for phenomenology, however, it is much easier to use a nonconserved local (or simple one-link point-split) vector current than the conserved one [6-8]. This must then be renormalized.

Renormalization schemes for nonconserved currents that make use (not necessarily explicitly) of ratios of matrix elements for conserved and nonconserved vector currents have a special status because nonperturbative contributions from higher-dimension operators are suppressed by powers of $a^{2}$. They give renormalization constants, $Z_{V}$, for nonconserved lattice vector currents that are exact in the $a \rightarrow 0$ limit. Such a $Z_{V}$ can then be combined with a matrix element of that nonconserved current in the lattice QCD calculation and the result extrapolated to zero lattice spacing. The same answer will be obtained in that limit with any such $Z_{V}$.

Following the discussion of perturbative matching earlier we can think of an exact $Z_{V}$ as consisting of a perturbative series in $\alpha_{s}$ that depends on the form of the vector current (and also in principle on terms arising from small instantons [9] or other nonperturbative effects of this kind) plus discretization effects that depend on the scheme and vanish as $a \rightarrow 0$ [10]. Note that we do not need to know what the perturbative series is; the method is completely nonperturbative. Which exact $Z_{V}$ to use is then simply an issue of numerical cost to achieve a given uncertainty and/or convenience.

One standard exact method for renormalizing nonconserved vector currents in lattice QCD is to require (electric) charge conservation i.e., that the vector form factor between identical hadrons at zero momentum transfer should have value 1 . Since this result would be obtained for the conserved current, $Z_{V}$ is implicitly a ratio of nonconserved to conserved current matrix elements between the two hadrons. This method is numerically fairly costly because it requires the calculation of two-point and three-point correlation functions. It can give numerically accurate results $[\mathcal{O}(0.1 \%)$ uncertainties] when averaged over a sufficiently large sample (hundreds) of gluon-field configurations. As above, we expect the $Z_{V}$ determined from this method [which we will denote $Z_{V}(\mathrm{~F}(0))$ ] to be equal to a perturbative matching factor up to discretization effects. This was tested by the HPQCD Collaboration in Appendix B of Ref. [11] for the local vector current made of HISQ quarks. Values for $Z_{V}^{\operatorname{loc}}(\mathrm{F}(0))$ were calculated at multiple values of the lattice spacing and gave a good fit to a perturbative expansion in $\alpha_{s}$ plus discretization effects, constraining the $\mathcal{O}\left(\alpha_{s}\right)$ coefficient to have the known value determined in lattice QCD perturbation theory.

Alternative methods of determining renormalization factors by defining a variety of momentum-subtraction schemes on the lattice [12-15] can produce precise results for $Z$ factors at lower computational cost. However, only some of these schemes are exact for $Z_{V}$ in the sense defined above.

The momentum-subtraction schemes define $Z_{V}$ from the ratio of two matrix elements calculated between external 
quark states of large virtuality, $\mu^{2}$, in a fixed gauge. Working at large $\mu^{2}$ is part of the definition of these schemes because nonperturbative contributions from higher-dimension operators will in general be suppressed by powers of $\mu^{2}$ and not $a^{2}$ as above. A wave-function renormalization factor is determined from the quark propagator. A vertex renormalization factor comes from an amputated vertex function for the vector current, on which momentum-subtraction renormalization conditions have been imposed. $Z_{V}$ is then obtained as the ratio of these two factors, with tiny statistical errors from a handful of gluon-field configurations if "momentum sources" are used [16].

The momentum-subtraction scheme known as RI-SMOM [15] is constructed around the WardTakahashi identity and so designed to give $Z_{V}=1$ for the lattice conserved current. We show explicitly that this is true for the HISQ action. This means that implementing the RI-SMOM scheme for nonconserved currents is equivalent to taking a ratio of vector vertex functions for conserved and nonconserved currents. We compare the $Z_{V}$ values obtained in the RI-SMOM scheme, $Z_{V}(\mathrm{SMOM})$, to those from the form factor method for the local vector HISQ current. We are able to show that, as expected, $Z_{V}^{\text {loc }}(\mathrm{SMOM})$ differs from $Z_{V}^{\text {loc }}(F(0))$ only by discretization effects so that the two methods will give the same answer for physical matrix elements in the continuum limit.

A popular momentum-subtraction scheme that does not make use of the vector Ward-Takahashi identity is the $\mathrm{RI}^{\prime}$-MOM scheme $[12,13]$. We show that in this scheme the $Z_{V}$ values for both the conserved and local vector currents are not exact but have contamination from nonperturbative (condensate) artifacts that survive the continuum limit. To make use of this scheme $Z_{V}$ must be redefined to use instead a ratio of the vector vertex function for conserved and nonconserved currents. We show the results from implementing this method.

We stress here that we are determining $Z_{V}$ very precisely and hence comparing values with uncertainties at the $0.1 \%$ level. Previous work has compared values for $Z_{V}$ for nonconserved currents from methods that use Ward identities and the RI'-MOM scheme (e.g., Refs. [17,18]) and concluded that there was agreement at the $1 \%$ level. Our more accurate results show clear disagreement, most obviously in the analysis for the conserved current.

Our earlier argument that $0.1 \%$ accuracy is needed for renormalization constants in pure lattice QCD can be extended when we study the impact of adding QED effects. When we allow the valence quarks to have electric charge (i.e., adding quenched QED to lattice QCD) we see a tiny impact (less than $0.1 \%$ ) on $Z_{V}$ using the HISQ action. We can now quantify and analyze this effect using the RI-SMOM scheme, having established that the nonperturbative $Z_{V}$ values behave correctly.
The paper is laid out as follows. We first discuss in Sec. II the exact lattice vector Ward-Takahashi identity that gives the conserved vector current for the HISQ action. We then give a brief overview of the momentum-subtraction schemes, called RI-SMOM and RI'-MOM, that we will use (abbreviating the names to SMOM and MOM) in Sec. III and, following that, a brief description of our lattice setup in Sec. IV. We show how the Ward-Takahashi identity works for the HISQ action in Sec. IVA so that the conserved current is not renormalized. This is then translated into the RI-SMOM scheme in Sec. IV B where $Z_{V}=1$ is obtained for the conserved current at all $a$ and $\mu$ values. For RI'MOM, however, condensate contributions are clearly evident in the $Z_{V}$ values for the conserved current as shown in Sec. IV C. In Secs. IV D and IV E we demonstrate the impact of the protection from the Ward-Takahashi identity on the renormalization factors for the simple local vector current $\left(\bar{\psi} \gamma \gamma_{\mu} \psi\right.$ with the fields at the same space-time point). The difference between $Z_{V}^{\text {loc }}(\mathrm{SMOM})$ and $Z_{V}^{\text {loc }}(\mathrm{F}(0))$ is purely a discretization effect; in the $\mathrm{RI}^{\prime}$-MOM scheme we demonstrate how to achieve the same outcome with a renormalization factor that is a ratio between that for the local and conserved currents. In Sec. V we show the impact of quenched QED on the $Z_{V}$ values obtained for the local current in the RI-SMOM scheme and compare to our expectations based on the work in earlier sections. Finally, in Sec. VI we discuss the implications of these results for ongoing and future calculations and give our conclusions. A similar picture to that for the local current is seen for the one-link point-split vector current and we give the RI-SMOM results for this case in Appendix B.

We reiterate the shorthand notation that we will use for the renormalization constants for clarity. $Z_{V}^{\mathrm{x}}(\mathrm{A})$ renormalizes the lattice vector current $x$ (cons, loc, 1link) to match the continuum current (in e.g., $\overline{\mathrm{MS}}$ ) and has been calculated in the scheme A $(\mathrm{F}(0), \mathrm{SMOM}, \mathrm{MOM})$.

\section{THE VECTOR WARD-TAKAHASHI IDENTITY ON THE LATTICE}

For both continuum and lattice [19,20] actions the derivation of the vector Ward-Takahashi identity proceeds from the observation that the path integral is invariant under a local change of the fermion field variables $\psi$ and $\bar{\psi}$ (only) that has unit Jacobian. Then

$$
\int \mathcal{D} \psi \mathcal{D} \bar{\psi} e^{-S\left[\psi^{e}, \bar{\psi}^{\epsilon}\right]} f\left(\psi^{\epsilon}, \bar{\psi}^{\epsilon}\right)=\langle f(\psi, \bar{\psi})\rangle .
$$

An example of such a transformation is to multiply $\psi$, say at point $x$, by a phase $e^{i \epsilon}$ and $\bar{\psi}(x)$ by $e^{-i \epsilon}$ :

$$
\psi(z) \rightarrow \psi^{\epsilon}(z) \equiv \begin{cases}e^{i \epsilon} \psi(x) & \text { for } z=x, \\ \psi(x) & \text { for } z \neq x\end{cases}
$$


Expanding Eq. (1) to first order in $\epsilon$ and denoting $\Delta X=$ $X^{\epsilon}-X$ to this order gives

$$
\langle-\Delta S \cdot f+\Delta f\rangle=0 .
$$

If we consider the path integral for the two point correlator $\left\langle\bar{\psi}\left(y_{1}\right) \psi\left(y_{2}\right)\right\rangle$ then $\Delta f$ becomes the difference of propagators from the points $y_{1}$ and $y_{2}$ to $x . \Delta S$ can be recast into the form $\Delta_{\mu} J^{\mu}$, allowing us to identify the conserved current $J$ associated with $S$. We have

$$
\begin{aligned}
\left\langle\Delta_{\mu} J^{\mu}(x) \bar{\psi}\left(y_{1}\right) \psi\left(y_{2}\right)\right\rangle= & \delta_{y_{2}, x}\left\langle\bar{\psi}\left(y_{1}\right) \psi(x)\right\rangle \\
& -\delta_{y_{1}, x}\left\langle\bar{\psi}(x) \psi\left(y_{2}\right)\right\rangle .
\end{aligned}
$$

The right-hand side is zero unless $y_{1}$ or $y_{2}$ overlaps with $x$ (and not with each other). Note that $\Delta_{\mu} J^{\mu}(x)$ is centred on the point $x$.

On the lattice $\Delta_{\mu}$ can be a simple forward $\left(\Delta_{\mu}^{+}\right)$or backward $\left(\Delta_{\mu}^{-}\right)$finite difference over one link. The current $J^{\mu}$ must then be chosen appropriately so that

$$
\begin{aligned}
\Delta^{\mu} J_{\mu}(x) & \equiv \Delta^{\mu,+} J_{\mu}^{-}=\sum_{\mu}\left(J_{\mu}^{-}(x+\hat{\mu})-J_{\mu}^{-}(x)\right) \\
& \equiv \Delta^{\mu,-} J_{\mu}^{+}=\sum_{\mu}\left(J_{\mu}^{+}(x)-J_{\mu}^{+}(x-\hat{\mu})\right) .
\end{aligned}
$$

We give $J_{\mu}^{+}$for the HISQ action [4] that we use in Appendix A. As discussed in the Introduction, it is rather complicated. It contains a number of three-link terms because of the Naik term [3] that removes tree-level $a^{2}$ errors in the action. The position-space Ward-Takahashi identity of Eq. (4) provides a test of the implementation of the conserved current and we have checked that this works for our implementation exactly on a single gluon-field configuration for a variety of choices of $y_{1}$ and $y_{2}$.

We can perform the exact Fourier transform on the lattice of Eq. (4). The left-hand side becomes

$$
\begin{aligned}
& \left(1-e^{i a q_{\mu}}\right) \int d^{4} x d^{4} y_{1} d^{4} y_{2} e^{i q x} e^{-i p_{1} y_{1}} e^{i p_{2} y_{2}} \\
& \quad \times\left\langle J^{\mu,+}(\tilde{x}) \bar{\psi}\left(y_{1}\right) \psi\left(y_{2}\right)\right\rangle
\end{aligned}
$$

where $a$ is the lattice spacing and we take $q=p_{1}-p_{2} . \tilde{x}$ is the midpoint of the link between $x$ and $x+\hat{\mu}$. The righthand side becomes

$$
\begin{aligned}
& \int d^{4} x d^{4} y_{1} e^{i p_{1} x} e^{-i p_{1} y_{1}}\left\langle\bar{\psi}\left(y_{1}\right) \psi(x)\right\rangle \\
& -\int d^{4} x d^{4} y_{2} e^{-i p_{2} x} e^{i p_{2} y_{2}}\left\langle\bar{\psi}(x) \psi\left(y_{2}\right)\right\rangle \\
& \equiv S\left(p_{1}\right)-S\left(p_{2}\right)
\end{aligned}
$$

where $S$ is the quark propagator. Then, multiplying both sides by the product of inverse quark propagators we reach the lattice version of the standard expression for the WardTakahashi identity,

$\frac{-2 i}{a} \sin \left(\frac{a q_{\mu}}{2}\right) \Lambda_{V}^{\mu,+}\left(p_{1}, p_{2}\right)=-S^{-1}\left(p_{1}\right)+S^{-1}\left(p_{2}\right)$.

$\Lambda_{V}^{\mu,+}$ is the amputated vertex function for the vector current $J^{\mu,+}$ (absorbing a factor of $e^{i a q_{\mu} / 2}$ into the vertex function since $J^{\mu,+}$ sits on a link). This equation is exact, gluon-field configuration by configuration, in lattice QCD and we will demonstrate this for the HISQ action in Sec. IVA.

As is well known, Eq. (8) tells us that any rescaling of the vertex by renormalization on the left-hand side has to match rescaling of the inverse propagators on the righthand side. This means that $J^{\mu,+}$ is not renormalized, i.e., that the renormalization factor for this conserved current, $Z_{V}^{\text {cons }}=1$. Since this is also true for the conserved current in the continuum $\overline{\mathrm{MS}}$ scheme then the matrix elements of the lattice conserved current will agree in the continuum limit with those in the $\overline{\mathrm{MS}}$ scheme.

A renormalized nonconserved vector current, written for example as $Z_{V}^{\text {loc }} V^{\text {loc, } \mu}$ for a local current, obeys the same equations as $J^{\mu,+}$ since it is by definition the same operator up to discretization effects on the lattice [10]. For the HISQ action

$$
J_{\mu}^{+}=Z_{V}^{\mathrm{loc}} V_{\mu}^{\mathrm{loc}}+\mathcal{O}\left(a^{2}\right) .
$$

Again this is well known, but we point it out here because it has implications for the accuracy of the determination of $Z_{V}^{\text {loc }}$ on the lattice. It means that, if $Z_{V}^{\text {loc }}$ is determined by a procedure which uses the Ward-Takahashi identity and gives 1 for the renormalization of $J^{\mu, \pm}$, then $Z_{V}^{\text {loc }}$ must be free of systematic errors from nonperturbative (condensate) artifacts in the continuum limit because these must cancel between the left- and right-hand sides of Eq. (8). $Z_{V}^{\text {loc }}$ can in principle be determined by substituting $Z_{V}^{\text {loc }} V^{\text {loc }}$ into the left-hand side of Eq. (8) for any $p_{1}$ and $p_{2}$. Hadronic matrix elements of $Z_{V}^{\text {loc }} V^{\text {loc }}$ will then differ from the results in the continuum purely by discretization effects (which will depend on $p_{1}$ and $p_{2}$ ) that can be extrapolated away straightforwardly using results at multiple values of the lattice spacing. The $Z_{V}$ so obtained is completely nonperturbative.

Using Eq. (8) in its full generality is unnecessarily complicated and there are lattice QCD methods that make use of it in specific, and simpler, kinematic configurations.

As $q \rightarrow 0$ the identity of Eq. (8) can be used to show that the vector form factor for the conserved current between quark or hadron states of the same momentum will be unity. The inverse of the vector form factor at the same kinematic point for a nonconserved current then gives its $Z_{V}$ value. This method clearly satisfies the criteria above for an exact determination of $Z_{V}$. 
We now discuss momentum-subtraction renormalization schemes on the lattice and the extent to which they make use of Eq. (8).

\section{MOMENTUM-SUBTRACTION SCHEMES USED ON THE LATTICE}

Momentum-subtraction schemes are useful intermediate schemes between the lattice regularization and the continuum $\overline{\mathrm{MS}}$ scheme in which it is now standard to quote results for scheme-dependent quantities. If the same momentum-subtraction scheme is implemented both in lattice QCD and in continuum QCD then the continuum limit of the lattice results will be in the continuum momentumsubtraction scheme (and should be independent of lattice action at that point). They can then be converted to the $\overline{\mathrm{MS}}$ scheme using continuum QCD perturbation theory.

A momentum-subtraction scheme imposes renormalization conditions on matrix elements between (in the cases we consider) external quark states so that the tree-level result is obtained, i.e., $Z_{\Gamma}$ is defined by

$$
Z_{\Gamma}\left\langle p_{1}\left|O_{\Gamma}\right| p_{2}\right\rangle=\left\langle p_{1}\left|O_{\Gamma}\right| p_{2}\right\rangle_{\text {tree }}
$$

for some operator $O_{\Gamma}=\bar{\psi} \Gamma \psi$, and $\left\langle p_{1}\right|$ and $\left|p_{2}\right\rangle$ external quark states with momenta $p_{1}$ and $p_{2}$, typically taken to have large magnitude. This calculation must of course be done in a fixed gauge, and this is usually taken to be Landau gauge, which can be straightforwardly implemented in lattice QCD. Effects from the existence of Gribov copies under the gauge fixing could arise in general; here we show that there are no such effects for $Z_{V}$ determined using the Ward-Takahashi identity.

Here we will concentrate on the RI-SMOM scheme $[14,15]$. This scheme uses a symmetric kinematic configuration with only one scale so that $p_{1}^{2}=p_{2}^{2}=q^{2}=\mu^{2}$ (where $q=p_{1}-p_{2}$ ). The wave-function renormalization is defined (using continuum notation) by

$$
Z_{q}=-\frac{i}{12 p^{2}} \operatorname{Tr}\left(\not p S^{-1}(p)\right) .
$$

The vector current renormalization follows from requiring

$$
\frac{Z_{V}}{Z_{q}} \frac{1}{12 q^{2}} \operatorname{Tr}\left(q_{\mu} \Lambda_{V}^{\mu}\left(p_{1}, p_{2}\right) \not q\right)=1 .
$$

The traces here are over spin and color and normalizations are chosen so that $Z_{q}=Z_{V}=1$ at tree level. The equations above are given for the continuum SMOM scheme. On the lattice we must take care to define the appropriate discretization for $q_{\mu}$ and $q^{2}$ in the various places that they appear. Below we will see what form $q_{\mu}$ must take in combination with the vertex function for the conserved current.

The RI-SMOM scheme was defined with the vector Ward-Takahashi identity in mind [15]. This reference shows how the identity defines the projectors needed for the vector vertex function in the continuum [given in Eq. (12)] so that $Z_{V}=1$ for the conserved current. Here we repeat this exercise, but now on the lattice. Returning to the Ward-Takahashi identity in Eq. (8) we can multiply both sides by $\not{q}$ and take the trace while dividing by $\hat{q}^{2}$ (where $\hat{q}$ is a discretization of $q$ to be defined later). This gives

$$
\begin{aligned}
& \frac{1}{12 \hat{q}^{2}} \frac{-2 i}{a} \sin \left(a q_{\mu} / 2\right) \operatorname{Tr}\left(\Lambda_{V}^{\mu,+} \not{q}\right) \\
& \quad=\frac{1}{12 \hat{q}^{2}}\left[-\operatorname{Tr}\left(S^{-1}\left(p_{1}\right) \hat{q}\right)+\operatorname{Tr}\left(S^{-1}\left(p_{2}\right) \tilde{q}\right)\right] .
\end{aligned}
$$

We can simplify the right-hand side assuming that the inverse propagator takes the general form $S^{-1}(p)=$ $i \not p \Sigma_{V}\left(p^{2}\right)+\Sigma_{S}\left(p^{2}\right)$ in the continuum (from relativistic invariance). Then, for the SMOM kinematics,

$$
\operatorname{Tr}\left(S^{-1}\left(p_{1}\right) \not\right)-\operatorname{Tr}\left(S^{-1}\left(p_{2}\right) \not q\right)=\operatorname{Tr}\left(S^{-1}(q) \not q\right) .
$$

On the lattice this formula could be broken by discretization effects. We do not see noticeable effects of this kind with the HISQ action, however, as we will discuss in Sec. IV B.

Using Eq. (14) in Eq. (13) and multiplying by $i$ then gives, from the Ward-Takahashi identity

$\frac{1}{12 \hat{q}^{2}} \frac{2}{a} \sin \left(a q_{\mu} / 2\right) \operatorname{Tr}\left(\Lambda_{V}^{\mu,+} \not{q}\right)=-\frac{i}{12 \hat{q}^{2}} \operatorname{Tr}\left(S^{-1}(q) \not{q}\right)$.

From Eq. (11) we see that the right-hand side of this expression is $Z_{q}$ in the RI-SMOM scheme. Comparing the left-hand side to Eq. (12) we see that this is $Z_{q} / Z_{V}^{\text {cons }}$ in the RI-SMOM scheme where $Z_{V}^{\text {cons }}$ is the $Z_{V}$ factor for the conserved current and the Ward-Takahashi identity requires us to discretize $q_{\mu}$ as $2 \sin \left(a q_{\mu} / 2\right) / a$ [ $\hat{q}$ is defined in Eq. (21)]. Then, from Eq. (15), we expect that $Z_{V}^{\text {cons }}(\mathrm{SMOM})=1$ on the lattice and no further renormalization is needed to match to $\overline{\mathrm{MS}}$. Notice that this works for any value of $q$.

We will show by explicit calculation that $Z_{V}^{\text {cons }}(\mathrm{SMOM})=1$ for the HISQ action in Sec. IV B. This is not true configuration by configuration, however. It does require an average over gluon fields.

Another popular momentum-subtraction scheme is RI'-MOM [12,13], abbreviated here to MOM. In this scheme $Z_{q}$ is defined in the same way, by Eq. (11), but $Z_{V}$ is defined by a different projector for the vector vertex function and the kinematic configuration for the MOM case is $p_{1}=p_{2}=p$ so that $q=0$. Instead of Eq. (12) we have, in the MOM scheme,

$$
\frac{Z_{V}}{Z_{q}} \frac{1}{12} \operatorname{Tr}\left(\gamma_{\mu} \Lambda_{V}^{\mu}(p)\right)=1 .
$$


Since this scheme does not correspond to a Ward-Takahashi identity, $Z_{V}$ determined this way needs further renormalization to match to the $\overline{\mathrm{MS}}$ scheme. More problematically, as we will show in Sec. IV, $Z_{V}^{\text {cons }}(\mathrm{MOM})$ for the HISQ action is significantly different from 1 and is contaminated by nonperturbative condensate effects.

The RI-SMOM $\gamma_{\mu}$ scheme [15] is similar to RI'-MOM above but uses the SMOM kinematics with $p_{1}^{2}=p_{2}^{2}=q^{2}$.

To calculate the renormalization constants for nonconserved currents we must combine the calculation of the vector vertex function for that current [Eq. (12) and appropriate modifications of it as described in the text] with the calculation of the wave-function renormalization [Eq. (11)]. We describe the results for the HISQ local vector current in the SMOM scheme in Sec. IV D. We are able to show that the renormalization factor for the local vector current in the SMOM scheme differs from that using the form factor method purely by discretization effects, demonstrating that it is an exact form of $Z_{V}$. The discretization effects depend on $q$ but the method is exact for any $q$; this is in contrast to the usual idea of a "window" of $q$ values to be used in momentum-subtraction schemes on the lattice [12].

The RI'-MOM scheme is not exact, as discussed above. We show in Sec. IV E that a modification of the method (reverting to one of the original suggestions in Ref. [12]) does, however, give an exact $Z_{V}$.

There are technical issues associated with implementing momentum-subtraction schemes for staggered quarks that we will not discuss here. We use the techniques developed in Ref. [21] and summarized again in Ref. [2] in the context of the RI-SMOM scheme. We will only discuss here specific issues that arise in the context of the vector current renormalization.

\section{THE LATTICE QCD CALCULATION}

We perform calculations on $n_{f}=2+1+1$ gluon-field configurations generated by the MILC Collaboration $[22,23]$ listed in Table I. These ensembles use an improved gluon action which removes discretization errors through $\mathcal{O}\left(\alpha_{s} a^{2}\right)$ [24]. They include the effect of $u / d, s$ and $c$ quarks in the sea using the HISQ action [4].

All gauge field configurations used are numerically fixed to Landau gauge by maximizing the trace over the gluonfield link with a gauge-fixing tolerance of $\epsilon=10^{-14}$. This is enough to remove the difficulties related to loose gauge fixing discussed in Ref. [2].

We use broadly the same calculational setup as in Ref. [2] but here we are considering vector current vertex functions rather than scalar ones. To implement momentum-subtraction schemes for staggered quarks we need to use momenta within a reduced Brillouin zone [21]

$$
-\pi / 2 \leq a p \leq \pi / 2
$$

For each momentum $a p_{1}$ or $a p_{2}$ we then calculate propagators or vertex functions with 16 copies of that momentum, $a p_{1}+\pi A$ and $a p_{2}+\pi B$ where $A$ and $B$ are four-vectors composed of 0 's and 1's. This then enables us to do the traces over spin for specific "tastes" of vector current implied by equations such as Eq. (15). There is also a trace over color in this equation so the $S^{-1}(q)$ factor on the right-hand side, for example, is actually a $48 \times 48$ matrix. Where necessary we will use the notation of Ref. [21] to denote specific spin tastes. As an example $\overline{\overline{\gamma_{\mu} \otimes I}}$ is the $16 \times 16$ matrix of 0 's and 1's that projects onto a taste-singlet vector in $A B$ space.

Twisted boundary conditions are utilized to give the incoming and outgoing quarks arbitrary momenta [28,29]. For the SMOM kinematics we take, with ordering $(x, y, z, t)$,

$$
\begin{aligned}
& a p_{1}=(a \mu, 0, a \mu, 0) / \sqrt{2}, \\
& a p_{2}=(a \mu,-a \mu, 0,0) / \sqrt{2} .
\end{aligned}
$$

For the MOM kinematics we take $a p_{2}=a p_{1}$. A range of $a \mu$ values are chosen at each lattice spacing, satisfying

TABLE I. Simulation parameters for the MILC gluon-field ensembles that we use, labeled by Set number in the first column. $\beta=10 / g^{2}$ is the bare QCD coupling and $w 0 / a$ gives the lattice spacing [25], using $w_{0}=$ $0.1715(9) \mathrm{fm}$ [26] determined from the $\pi$ meson decay constant, $f_{\pi}$. Note that, for each group of ensembles at a given value of $\beta$ we use the $w_{0} / a$ value corresponding to the physical sea quark mass limit [2], using results from Ref. [27]. $L_{s}$ and $L_{t}$ give the lattice dimensions. $a m_{l}^{\text {sea }}, a m_{s}^{\text {sea }}$ and $a m_{c}^{\text {sea }}$ give the sea quark masses in lattice units. Set 1 will be referred to in the text as "very coarse," Sets $2-5$ as "coarse," Set 6 as "fine" and Set 7 as "superfine."

\begin{tabular}{lccccccc}
\hline Set & $\beta$ & $w_{0} / a$ & $L_{s}$ & $L_{t}$ & $a m_{l}^{\text {sea }}$ & $a m_{s}^{\text {sea }}$ & $a m_{c}^{\text {sea }}$ \\
\hline 1 & 5.80 & $1.1322(14)$ & 24 & 48 & 0.00640 & 0.0640 & 0.828 \\
2 & 6.00 & $1.4075(18)$ & 24 & 64 & 0.0102 & 0.0509 & 0.635 \\
3 & 6.00 & $1.4075(18)$ & 24 & 64 & 0.00507 & 0.0507 & 0.628 \\
4 & 6.00 & $1.4075(18)$ & 32 & 64 & 0.00507 & 0.0507 & 0.628 \\
5 & 6.00 & $1.4075(18)$ & 40 & 64 & 0.00507 & 0.0507 & 0.628 \\
6 & 6.30 & $1.9500(21)$ & 48 & 96 & 0.00363 & 0.0363 & 0.430 \\
7 & 6.72 & $2.994(10)$ & 48 & 144 & 0.0048 & 0.024 & 0.286 \\
\hline \hline
\end{tabular}


Eq. (17). This allows us to reach $\mu$ values of $3 \mathrm{GeV}$ on coarse lattices and $4 \mathrm{GeV}$ on fine and superfine lattices [2]. The $\mu$ values can be tuned very accurately (to three decimal places).

Relatively small samples (20 configurations) give small statistical uncertainties for $Z_{V}$ at the $\mu$ values that we use (with momentum sources for the propagators). A bootstrap method is used to estimate all uncertainties and include correlations between results at different $\mu$ values on a given ensemble. Bootstrap samples are formed for each $Z_{q}$ and each $\Lambda_{V}$ and the bootstrap averages are then fed into the ratio to determine $Z_{V}$.

All of our results are determined at small but nonzero valence quark mass. Degenerate masses are used for the incoming and outgoing quarks (but note that there is no need for the calculation of disconnected contributions). As the momentum-subtraction schemes that we consider are in principle defined at zero valence quark mass (but direct calculation at this point will have finite-volume issues) it is necessary to calculate each $Z_{V}$ at different quark masses and then extrapolate to the $a m_{\mathrm{val}}=0$ point. To do this we perform all calculations at three masses corresponding to the light sea quark mass on a given ensemble, $a m_{l}$, and at $2 a m_{l}$ and $3 a m_{l}$. Dependence on $a m_{\mathrm{val}}$ can come from discretization effects and from the contribution of nonperturbative condensate terms.

We follow the procedure used for $Z_{m}$ in Ref. [2] and extrapolate $Z_{V}$ results using a polynomial in $a m_{\mathrm{val}} / a m_{s}$ :

$Z_{V}\left(a m_{\mathrm{val}}, \mu\right)=Z_{V}(\mu)+d_{1}(\mu) \frac{a m_{\mathrm{val}}}{a m_{s}}+d_{2}(\mu)\left(\frac{a m_{\mathrm{val}}}{a m_{s}}\right)^{2}$.

We find no need for higher powers of $a m_{\mathrm{val}} / a m_{s}$ here as the valence mass dependence of $Z_{V}$ is observed to be very mild in all cases. For the priors for the coefficients $d_{i}$ we use $\{0 \pm 0.1,0 \pm 0.01\}$ at $\mu=2 \mathrm{GeV}$ with the widths decreased according to $\mu^{-2}$.

Any sea quark mass dependence should be suppressed relative to the valence mass dependence by powers of $\alpha_{s}$ and this was observed in Ref. [2]. As the valence mass dependence is already negligible the sea mass dependence should be tiny here and we ignore it.

\section{A. The Ward-Takahashi identity on the lattice}

In this section we test the exact lattice Ward-Takahashi identity for HISQ quarks, i.e., Eq. (8). If we have correctly implemented the lattice conserved vector current, this equation is true as a $3 \times 3$ matrix in color space. It is also true for any $p_{1}$ and $p_{2}$ (except that it reduces to $0=0$ for $p_{1}=p_{2}$ ), any value of the quark mass and any gauge. We test it for the SMOM kinematic configuration of Eq. (18).

Figure 1 shows the results as a ratio of the difference of inverse propagators on the right-hand side of Eq. (8) to the

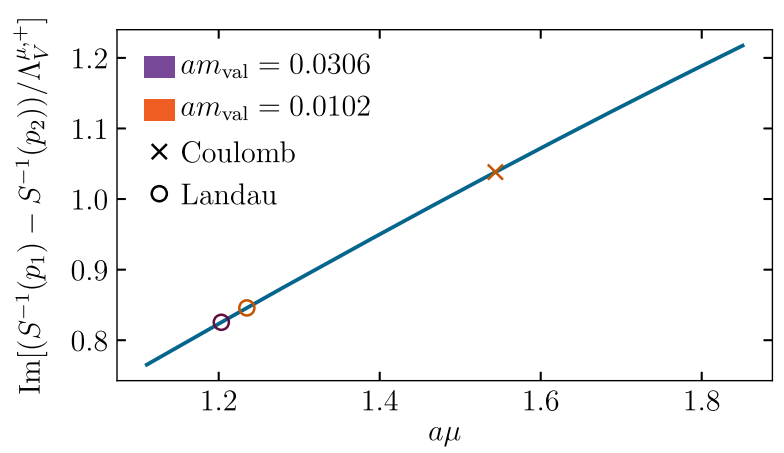

FIG. 1. Demonstration of the vector Ward-Takahashi identity in momentum space [Eq. (8)] for HISQ quarks on the lattice on a single gluon-field configuration from Set 2 . The plot shows the ratio of the right-hand side of this equation to the amputated vertex function for the conserved vector current on the left-hand side for the SMOM kinematic configuration, Eq. (18). This is a matrix equation and this plots shows the result of averaging over all matrix components (which agree) and the two nonzero components of $q_{\mu}$. The solid line is the value of $2 \sin \left(a q_{\mu} / 2\right)$ for a nonzero component of $q_{\mu}$. The points correspond to lattice results for the ratio on a single configuration with crosses giving Coulomb gauge-fixed results and the circles Landau gauge-fixed results. Orange points correspond to a valence mass of $a m_{\mathrm{val}}=$ 0.0306 while purple points correspond to 0.0102 . The WardTakahashi identity requires these points to lie on the line as they do.

amputated vertex function for the conserved vector current on the left-hand side. This is averaged over color components (which all agree) and summed over the two nonzero components of $q_{\mu}$ (which take the same value $a \mu / \sqrt{2}$ in each of the $y$ and $z$ directions for the SMOM kinematics). The Ward-Takahashi identity [Eq. (8)] requires this ratio to be exactly equal to $2 \sin [a \mu /(2 \sqrt{2})]$, which is plotted as the line.

The plot shows that this expectation works to high precision (double-precision accuracy here), on a single configuration taken as an example from Set 2. Results are given for three different $a \mu$ values with two different valence quark masses and in two different gauges. The agreement between the points and the line demonstrates the Ward-Takahashi identity working explicitly on the lattice for the conserved HISQ current of Eq. (A1). The agreement seen in two different gauges is evidence that the WardTakahashi identity works in any gauge, as it must, and therefore its operation is also independent of any Gribov copy issue in the gauge-fixing procedure.

\section{B. $Z_{V}$ for the conserved current in the RI-SMOM scheme}

To determine $Z_{V}$ for the HISQ conserved current in the RI-SMOM scheme we adapt Eqs. (11) and (12) to the case of staggered quarks on the lattice, as partly discussed already in Sec. III. For staggered quarks the inverse 


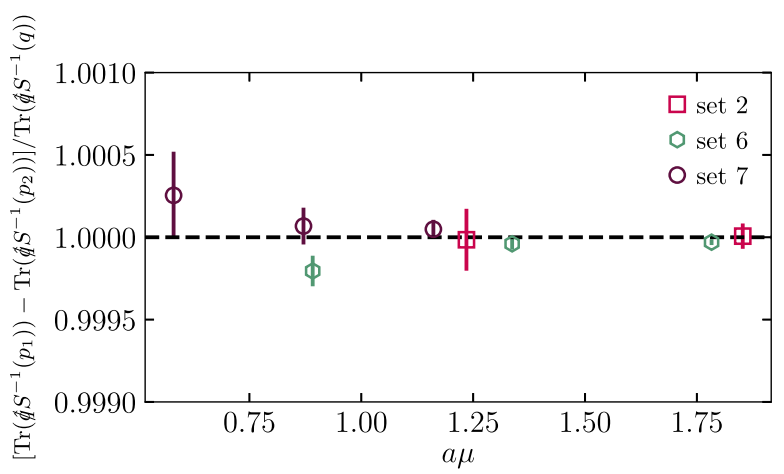

FIG. 2. A test of the expression for the difference of inverse propagators with momentum $p_{1}$ and $p_{2}$ in Eq. (14). We show results on coarse, fine and superfine lattices (Sets 2, 5 and 7) for a variety of $\mu$ values in lattice units where $\left|a p_{1}\right|=\left|a p_{2}\right|=$ $|a q|=a \mu$.

propagator is a taste singlet [21] and so the HISQ version of Eq. (11) is

$$
Z_{q}(q)=-\frac{i}{48} \sum_{\mu} \frac{a \hat{q}_{\mu}}{(a \hat{q})^{2}} \operatorname{Tr}\left[\overline{\overline{\left(\gamma_{\mu} \otimes I\right)}} S^{-1}(q)\right] .
$$

The trace is now over color and $A B$-space indices. $\hat{q}$ is given by

$$
a \hat{q}_{\mu}=\sin \left(a q_{\mu}\right)+\frac{1}{6} \sin ^{3}\left(a q_{\mu}\right) .
$$

This choice is dictated by the momentum-subtraction requirement that $Z_{q}$ should be 1 in the noninteracting (tree-level) case and the fact that the derivatives in the HISQ action are improved through $\mathcal{O}\left(a^{2}\right)$ [4]. Likewise the HISQ calculation for $Z_{V}$ for this case is given by

$$
\frac{Z_{q}(q)}{Z_{V}(q)}=\frac{1}{48} \sum_{\mu, \nu} 2 \sin \left(a q_{\mu} / 2\right) \frac{a \hat{q}_{\nu}}{(a \hat{q})^{2}} \operatorname{Tr}\left[\overline{\overline{\left(\gamma_{\nu} \otimes I\right)}} \Lambda_{V}^{\mu,+}\right]
$$

In Sec. III it was shown how the Ward-Takahashi identity leads to the exact expression of Eq. (13) on the lattice when the conserved current is used in the vertex function. In order to obtain $Z_{V}=1$ for the conserved current we also need Eq. (14) to be satisfied exactly. In Fig. 2 we give a test of this relationship. The figure shows the ratio of the difference of the two inverse propagators with momentum $p_{1}$ and $p_{2}$ to that of the propagator with momentum $q$, where the inverse propagators are multiplied by $\not{q}$ and the trace taken. We use $\hat{q}$ here [Eq. (21)] instead of simply $q$ to be consistent with what we used in the determination of $Z_{q}$ in Eq. (20) above. The results for the ratio plotted would be the same for $q$ as for $\hat{q}$. The results for the ratio in Fig. 2 are seen to be consistent with 1.0 to better than $0.05 \%$. The

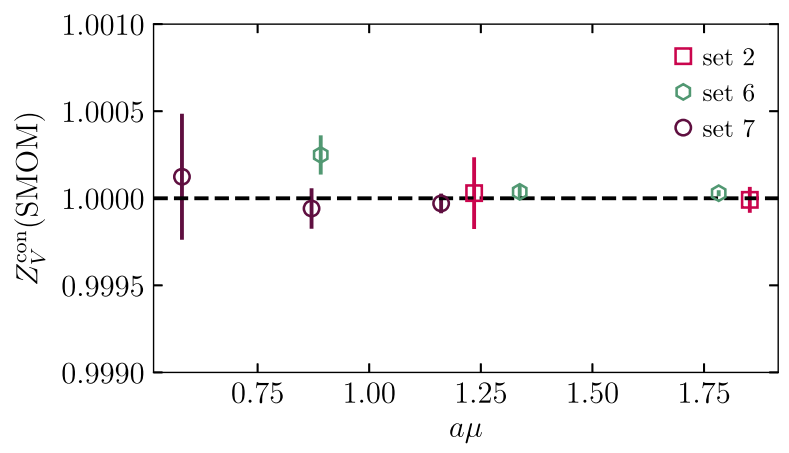

FIG. 3. The $Z_{V}$ value obtained for the conserved vector current in the RI-SMOM scheme on coarse, fine and superfine gluonfield configurations (Sets 2, 5 and 7). Values are given for a variety of $\mu$ values in lattice units where $\left|a p_{1}\right|=\left|a p_{2}\right|=$ $|a q|=a \mu$.

statistical uncertainties plotted are from a bootstrap over results from 20 gluon-field configurations.

Figure 2 shows that discretization effects in the HISQ action have no effect on Eq. (14) at the level of accuracy to which we are working. There are no tree-level $a^{2}$ errors with HISQ [4] and there is a U(1) axial symmetry; both of these constrain the form that discretization effects can take [21]. A further constraint comes from the form for the $p_{1}$ and $p_{2}$ momenta (and $q$ ) to achieve the SMOM kinematics. Each has only two nonzero momentum components, as shown in Eq. (18). This means, for example, that discretization errors in $S^{-1}$ containing three different $\gamma$ matrices and associated momenta are zero.

Figure 3 shows the resulting $Z_{V}$ value obtained for the conserved vector current in the RI-SMOM scheme, combining the results from Eqs. (22) and (20) and performing the extrapolation to zero quark mass as described in Sec. IV (this has very little impact). The value obtained for $Z_{V}$ for the conserved current is 1 to better than $0.05 \%$ at all $\mu$ values. Fitting the results shown in Fig. 3 to a constant value of 1.0 returns a $\chi^{2} /$ d.o.f. of 1.3 for eight degrees of freedom $(Q=0.26)$.

\section{C. $Z_{V}$ for the conserved current in the $R I^{\prime}$-MOM scheme}

We now turn to the renormalization of the conserved current in the standard $\mathrm{RI}^{\prime}-\mathrm{MOM}$ scheme where a very different picture emerges.

The kinematic conditions in the MOM scheme are that the incoming and outgoing quark fields for the vertex function should have the same momentum, $a p_{1}=a p_{2}$ so that $a q=0$. We will denote this momentum by $a p$ with $|a p|=a \mu$. We take the form of $a p$ to be that of $a p_{1}$ in the SMOM scheme [Eq. (18)]. To implement the RI'-MOM scheme we determine the wave-function renormalization, $Z_{q}(p)$, in the same way as for the RI-SMOM scheme using Eq. (20). To determine $Z_{V}$ we use 


$$
\frac{Z_{q}}{Z_{V}}=\frac{1}{48} \frac{1}{V_{\gamma \otimes I}^{\text {cons }}} \sum_{\mu} \operatorname{Tr}\left[\overline{\overline{\left(\gamma_{\mu} \otimes I\right)}} \Lambda_{V}^{\mu,+}\right] .
$$

This uses the $\mathrm{RI}^{\prime}-\mathrm{MOM}$ vector vertex projector, which is simply $\gamma_{\mu}$ [see Eq. (16)], expressed here in the appropriate taste-singlet form for implementation with staggered quarks. Because the conserved current is a point-split operator the tree-level vertex function is not simply 1 . We therefore need to divide by the tree-level matrix element for the conserved current that we denote here as $V_{\gamma_{\mu} \otimes I}^{\text {cons }}$. How to calculate these tree-level factors is discussed in Ref. [21]. We have

$V_{\gamma \otimes I}^{\mathrm{cons}}=\prod_{\mu}\left(\frac{9}{8} \cos \left(a p_{\mu}(S-T)_{\mu}\right)-\frac{1}{8} \cos \left(3 a p_{\mu}(S-T)_{\mu}\right)\right)$.

The spin-taste four-vector $S-T$ is composed of 1's and 0's. For the taste-singlet vector it takes the value 1 for component $\mu$ and 0 otherwise. So the only components of the product that do not take the value 1 are those for component $\mu$ that matches the direction of the current, provided that $a p$ has a nonzero component in that direction.

Because the RI'-MOM scheme is not based on the WardTakahashi identity $Z_{V}$ will not be 1 for the conserved current. This means that to reach the $\overline{\mathrm{MS}}$ scheme, even for the continuum $\mathrm{RI}^{\prime}-\mathrm{MOM}$ scheme, requires an additional renormalization factor. The renormalization factor that takes the lattice vector current to the continuum is then

$$
Z_{V}(\mathrm{MOM})=Z_{V}^{\overline{\mathrm{MS}} / \mathrm{RI}^{\prime}-\mathrm{MOM}^{\mathrm{MOM}} Z_{V}^{\mathrm{MOaw}} .}
$$

$Z_{V}^{\mathrm{MOM}, \text { raw }}$ is the raw renormalization factor calculated using Eq. (23) on the lattice. The factor $Z_{V}^{\overline{\mathrm{MS}} / \mathrm{MOM}}$ can be determined from the perturbative QCD expansions in the continuum for the conversion between RI'-MOM and RIMOM given in Ref. [13] (see Ref. [30] and the Appendix of Ref. [21]). The values needed for our $\mu$ values are given in Table II; they are all close to 1 since the expansion starts at $\mathcal{O}\left(\alpha_{s}^{2}\right)$.

Figure 4 shows our results for $Z_{V}$ for the conserved HISQ current obtained by implementing the RI'-MOM scheme on the lattice. We have converted the $Z_{V}$ to the value that takes the lattice results to the $\overline{\mathrm{MS}}$ scheme using Eq. (25). Results are shown, after extrapolation to zero valence quark mass, at a variety of $\mu$ values from 2 to $4 \mathrm{GeV}$ and at three different values of the lattice spacing. It is immediately clear that the values of $Z_{V}^{\text {cons }}(\mathrm{MOM})$ are not 1 . This is in sharp contrast to results in the RI-SMOM scheme where, as we showed in Sec. IV B, the value 1 is obtained. This result is shown by the black line at 1 in Fig. 4.

To understand the discrepancy from 1 for $Z_{V}^{\text {cons }}$ in the $\mathrm{RI}^{\prime}-\mathrm{MOM}$ case, we fit the points shown in Fig. 4
TABLE II. Conversion factors from the continuum RI'-MOM scheme to $\overline{\mathrm{MS}}$ at the $\mu$ values used in this calculation, calculated with $n_{\mathrm{f}}=4$ using the results of Ref. [13]. Results for $Z_{V}$ obtained on the lattice with the standard RI'-MOM approach must be multiplied by these values to give results in the $\overline{\mathrm{MS}}$ scheme in the continuum limit.

\begin{tabular}{ll}
\hline \hline$\mu[\mathrm{GeV}]$ & $Z_{V}^{\overline{\mathrm{MS}} / \mathrm{RI}^{\prime}-\mathrm{MOM}}$ \\
\hline 2 & $0.99118(38)$ \\
2.5 & $0.99308(26)$ \\
3 & $0.99420(20)$ \\
4 & $0.99549(14)$ \\
\hline \hline
\end{tabular}

(including the correlations between them) to a form that allows for both discretization effects and condensate contributions:

$$
\begin{aligned}
& Z_{V}^{\text {cons }}(\operatorname{MOM})(a, \mu) \\
& =1+\sum_{i=1}^{5} c_{a^{2} \mu^{2}}^{(i)}(a \mu / \pi)^{2 i} \\
& \quad+\sum_{i=1}^{5} c_{\alpha a^{2} \mu^{2}}^{(i)}(a \mu / \pi)^{2 i} \alpha_{\overline{\mathrm{MS}}}(1 / a)+c_{\alpha}\left(\alpha_{\overline{\mathrm{MS}}}(\mu) / \pi\right)^{4} \\
& \quad+\sum_{j=1}^{5} c_{\mathrm{cond}}^{(j)} \alpha_{\overline{\mathrm{MS}}}(\mu) \frac{(1 \mathrm{GeV})^{2 j}}{\mu^{2 j}} \times\left[1+c_{\mathrm{cond}, a^{2}}^{(j)}(a \Lambda / \pi)^{2}\right] .
\end{aligned}
$$

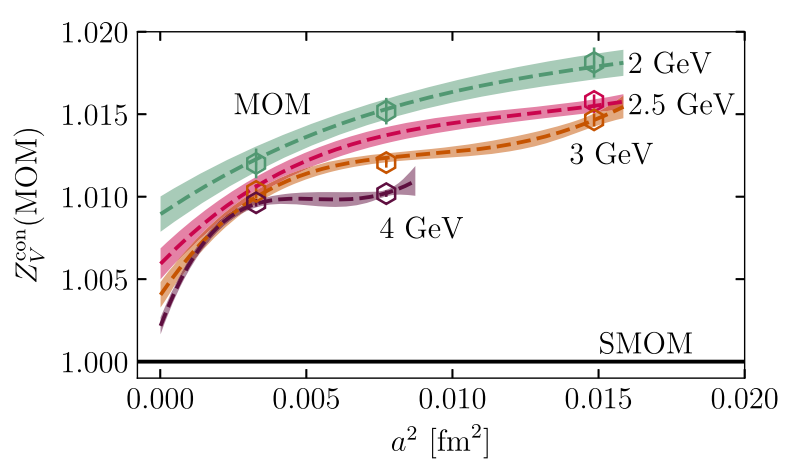

FIG. 4. Points labeled "MOM" show the renormalization factor for the HISQ conserved vector current which takes results from the lattice scheme to the $\overline{\mathrm{MS}}$ scheme obtained using a lattice calculation in the RI'-MOM scheme. These should be contrasted with results obtained using a lattice calculation in the RI-SMOM scheme which give a value of 1.0, shown as the black line labeled "SMOM." Points are given for $\mu$ values of 2, 2.5, 3 and $4 \mathrm{GeV}$, indicated by different colors, and on coarse, fine and superfine lattices. The fit shown [see Eq. (26)] accounts for discretization errors and condensate contributions, which prove to be necessary for a good fit. The separation of the results for different $\mu$ values in the continuum limit (at $a=0$ ) is a result of the condensate contributions that appear in $Z_{V}$ when calculated in the RI'-MOM scheme on the lattice. 
Note that this constrains $Z_{V}^{\text {cons }}(\mathrm{MOM})$ to be 1 in the continuum once condensates are removed. Here $\alpha_{\overline{\mathrm{MS}}}(\mu)$ is the value of the strong coupling constant in the $\overline{\mathrm{MS}}$ scheme at the scale $\mu$ calculated from running the value obtained in Ref. [27] using the four-loop QCD $\beta$ function. The fit allows for discretization errors of the generic form $(a \mu)^{2 i}$ and terms $\mathcal{O}\left(\alpha_{s}(a \mu)^{2 i}\right)$; only even powers of $a$ appear due to the remnant chiral symmetry of staggered quarks. Note that in principle we have removed $(a \mu)^{2}$ terms by dividing by $V_{\gamma \otimes I}^{\text {cons }}$; the fit returns only a small coefficient for this term. The $\alpha_{s}$-suppressed discretization terms are included as the very small statistical uncertainties on the results mean that these terms can have an effect on the fit. The fourth term allows for systematic uncertainty from the missing $\alpha_{s}^{4}$ term in the RI'-MOM-to- $\overline{\mathrm{MS}}$ conversion factor [Eq. (25)].

The condensate terms in the final line of Eq. (26) start at $1 / \mu^{2}$ to allow for the gauge-noninvariant $\left\langle A^{2}\right\rangle$ condensate present in the operator product expansion (OPE) of the quark propagator [31]. For the MOM kinematic setup it is not possible to perform an OPE for the vertex functions as they are not short-distance quantities $(q=0)$, so a complete analysis of what nonperturbative artifacts we expect to see in $Z_{V}$ is not possible. However, on general grounds we expect terms with inverse powers of $\mu$ to appear and we also allow these terms to have discretization effects. We include even inverse powers of $\mu$ up to $1 / \mu^{8}$.

We use a Bayesian fit approach [32] in which coefficients are constrained by priors with a Gaussian distribution of a given central value and width. All coefficients in the fit form of Eq. (26) are given priors of $0 \pm 1$, except for that of the $\left(\alpha_{s} / \pi\right)^{4}$ term which has prior $0 \pm 5$ based on the lower-order coefficients. The choices for the priors are based on reasonable values for the coefficients of the terms in the fit. For example, discretization effects are expected to appear as even powers of a physical scale (such as $\mu$ or $\Lambda$ here) divided by the ultraviolet cutoff $(\pi / a)$ with coefficients of order one.

The results of the fit are shown as the colored dashed lines in Fig. 4. The fit has a $\chi^{2} /$ d.o.f. of 0.6. It is already obvious from the figure that discretization effects are not the only source of the discrepancy in $Z_{V}$ from 1 . This is emphasized by attempting the fit without condensate terms [i.e., missing the last line of Eq. (26)]. Without the condensate terms the quality of the fit is very poor, with a $\chi^{2} /$ d.o.f. of 7.7 , in contrast to the fit of Eq. (26). The sizable contribution from the lowest-order condensate is reflected in the coefficient found by the fit of

$$
c_{\text {cond }}^{(1)}=0.154(54)
$$

The higher-order condensates cannot be pinned down by the fit.
The correct answer for $Z_{V}$ for the conserved current in the continuum limit is, of course, 1 . Our results and fit show that this can only be obtained from a calculation in the RI'MOM scheme by working at multiple $\mu$ values at multiple values of the lattice spacing and fitting as a function of $\mu$ and $a$ to identify and remove the condensate contributions. If this is not done, systematic errors of $\mathcal{O}(1 \%)$ (depending on the $\mu$ value) are present in $Z_{V}$, as is clear from Fig. 4 .

The issue will resurface when we discuss the use of the RI'-MOM scheme to renormalize nonconserved currents, specifically the HISQ local vector current, in Sec. IV E.

\section{D. $Z_{V}$ for the local current in the RI-SMOM scheme}

We now turn to the calculation of the renormalization constant for a nonconserved vector current using the RISMOM scheme. We will study the local current constructed from HISQ quarks since this is the simplest current and used in many analyses, such as the connected hadronic vacuum polarization contribution to the anomalous magnetic moment of the muon [33].

In Ref. [11] the renormalization constant for the HISQ local current was calculated using the form factor method discussed in Sec. II. Results are given for very coarse, coarse and fine lattices in Table IV of that reference. The calculation was done using valence $s$ quarks and the form factor was determined for the local temporal vector current between two $s \bar{s}$ pseudoscalar mesons at rest. ${ }^{1}$ From the discussion in Sec. II we expect such a determination of $Z_{V}$ to be exact so that $Z_{V}^{\text {loc }}(\mathrm{F}(0))$ is equal to a perturbative series in $\alpha_{s}$ that matches the lattice scheme to the $\overline{\mathrm{MS}}$ scheme, up to discretization effects. This was tested in Ref. [11] (Appendix B) by fitting the $Z_{V}$ results to this form, including the known $\mathcal{O}\left(\alpha_{s}\right)$ coefficient in the perturbative series. A good fit was obtained that allowed values for $Z_{V}^{\text {loc }}(\mathrm{F}(0))$ to be inferred on finer lattices. Here we will calculate $Z_{V}^{\text {loc }}(\mathrm{SMOM})$ and compare it to $Z_{V}^{\text {loc }}(\mathrm{F}(0))$. They should both contain the same perturbative series (since this is unique for a given operator) and differ only by discretization effects.

To calculate $Z_{V}^{\text {loc }}(\mathrm{SMOM})$ a little care is required in the construction of the SMOM vector vertex function with HISQ quarks. The operator $\phi_{1} q_{\mu} \Lambda_{V}^{\mu}$ of Eq. (12) must be constructed to be a taste singlet. For a local (in spin-taste notation, $\gamma_{\mu} \otimes \gamma_{\mu}$ ) current $\Lambda_{V}^{\mu}$ will have taste $\gamma_{\mu}$. This means that the $\not$ in the vertex function must also have this taste. The correct construction is

$$
\sum_{\mu, \nu} \hat{q}_{\nu}^{\overline{\left(\gamma_{\nu} \otimes \gamma_{\mu}\right)}} \hat{q}_{\mu} \Lambda_{V, \mathrm{loc}}^{\mu}
$$

\footnotetext{
${ }^{1}$ Note that the "spectator" quark used the clover formalism in this case, in order for the staggered tastes to cancel in the correlation function.
} 
TABLE III. Local vector current renormalization factors, $Z_{V}^{\text {loc }}$ for a variety of $\mu$ values (given in column 2) on gluon-field configurations at different lattice spacing values (denoted by the Set number in column 1). Column 3 gives results using the RI-SMOM scheme and column 4 gives results using the standard $\mathrm{RI}^{\prime}-\mathrm{MOM}$ scheme. Note that the RI'-MOM results include the additional renormalization factor of Eq. (25) (Table II) that is needed to take the lattice current all the way to the $\overline{\mathrm{MS}}$ scheme. Results are extrapolated to zero valence quark mass. Columns 5 and 6 give results for the modified (denoted by Rc) RI'-MOM and RI-SMOM $\gamma_{\mu}$ schemes in which a ratio to the value for the conserved current renormalization in that scheme has been taken [Eq. (32)].

\begin{tabular}{|c|c|c|c|c|c|}
\hline Set & $\mu[\mathrm{GeV}]$ & $Z_{V}^{\mathrm{loc}}(\mathrm{SMOM})$ & $Z_{V}^{\mathrm{loc}}(\mathrm{MOM})$ & $Z_{V}^{\text {loc }}\left(\mathrm{MOM}_{\mathrm{Rc}}\right)$ & $Z_{V}^{\mathrm{loc}}\left(\mathrm{SMOM}_{\gamma_{\mu}, \mathrm{Rc}}\right)$ \\
\hline 1 & 1 & $0.9743(11)$ & $\ldots$ & $\ldots$ & $\ldots$ \\
\hline 2 & 1 & $0.9837(20)$ & $\ldots$ & $\ldots$ & $\ldots$ \\
\hline 1 & 2 & $0.95932(18)$ & $\ldots$ & $\ldots$ & $\ldots$ \\
\hline 2 & 2 & $0.97255(22)$ & $0.98771(85)$ & $0.97012(25)$ & $0.91864(25)$ \\
\hline 6 & 2 & $0.98445(11)$ & $0.99784(79)$ & $0.98292(44)$ & $0.959434(58)$ \\
\hline 7 & 2 & $0.99090(36)$ & $1.00202(89)$ & $0.99012(19)$ & $0.982435(21)$ \\
\hline 2 & 2.5 & $0.96768(12)$ & $0.97968(34)$ & $0.96447(17)$ & $0.89506(19)$ \\
\hline 2 & 3 & $0.964328(75)$ & $0.97434(26)$ & $0.96027(23)$ & $0.87733(21)$ \\
\hline 6 & 3 & $0.977214(35)$ & $0.98785(28)$ & $0.97608(14)$ & $0.930025(40)$ \\
\hline 7 & 3 & $0.98702(11)$ & $0.99651(43)$ & $0.98633(11)$ & $0.969563(42)$ \\
\hline 6 & 4 & $0.972415(18)$ & $0.98090(16)$ & $0.971009(90)$ & $0.905823(40)$ \\
\hline 7 & 4 & $0.983270(54)$ & $0.99241(21)$ & $0.982942(40)$ & $0.954992(30)$ \\
\hline
\end{tabular}

Taking the spin-color trace of this operator and dividing by $48 \hat{q}^{2}$ then gives $Z_{q} / Z_{V}$. The wave-function renormalization is calculated in the same way as for the conserved current, Eq. (20). Results for $Z_{V}^{\mathrm{loc}}(\mathrm{SMOM})$ are given in Table III (column 3). This is after extrapolation to zero valence quark mass. Figure 5 shows that the impact of this is very small (we expect in this case that the mass dependence is purely a discretization effect).

Figure 6 (top plot) shows our results as a difference between $Z_{V}^{\text {loc }}(\mathrm{SMOM})$ and $Z_{V}^{\mathrm{loc}}(\mathrm{F}(0))$. $Z_{V}^{\text {loc }}(\mathrm{F}(0))$ values are from Ref. [11] and are obtained on the same gluon-field configurations that we use here. We plot the difference for the multiple $\mu$ values used for the $Z_{V}^{\text {loc }}(\mathrm{SMOM})$ determination as a function of lattice spacing in Fig. 6. Results are shown from very coarse to superfine lattice spacings noting that higher $\mu$ values are only accessible on finer lattices because of the constraint in Eq. (17).

We can readily fit this difference of $Z_{V}^{\text {loc }}$ values, $\Delta Z_{V}^{\text {loc }}$, to a function constructed from possible discretization effects.

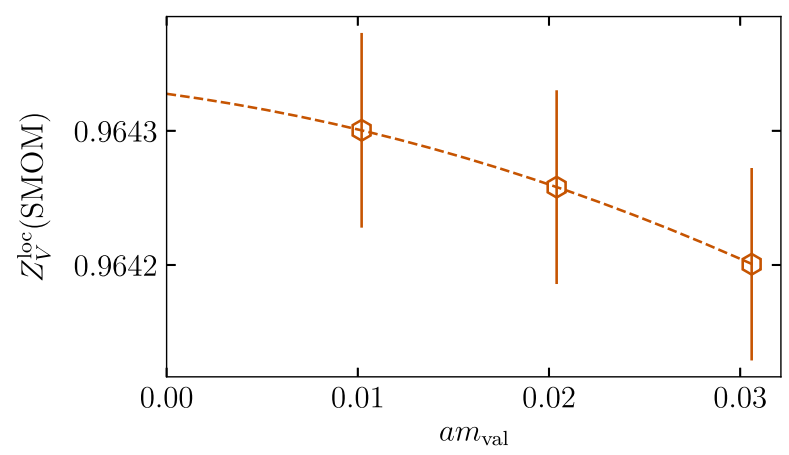

FIG. 5. Valence mass dependence of $Z_{V}^{\text {loc }}(\mathrm{SMOM})$ values obtained in the RI-SMOM scheme. Results and extrapolation are shown for $\mu=3 \mathrm{GeV}$ on Set 2 .
To keep the fit as general as possible we also allow for the existence of condensate terms to see to what extent they are constrained by the fit. We also allow for condensate terms multiplied by discretization effects that would vanish in the continuum limit (and are therefore benign). We use

$$
\begin{aligned}
\Delta Z_{V}^{\mathrm{loc}}(a, \mu)= & \sum_{i=1}^{3}\left[c_{a^{2} \mu^{2}}^{(i)}(a \mu / \pi)^{2 i}\right. \\
& \left.+c_{\alpha a^{2} \mu^{2}}^{(i)}(a \mu / \pi)^{2 i} \alpha \overline{\mathrm{MS}}(1 / a)\right] \\
& +\sum_{j=1}^{3} c_{\text {cond }}^{(j)} \alpha_{\overline{\mathrm{MS}}}(\mu) \frac{(1 \mathrm{GeV})^{2 j}}{\mu^{2 j}} \\
& \times\left[1+c_{\text {cond }, a^{2}}^{(j)}(a \Lambda / \pi)^{2}\right] .
\end{aligned}
$$

All coefficients are given priors $0 \pm 1$. This fit has a $\chi^{2} /$ d.o.f. value of 0.18 and finds no significant condensate contribution. The lowest-order $\left(1 / \mu^{2}\right)$ condensate term is constrained by the fit to have a very small coefficient compatible with zero: $-0.020(44)$ [cf. Eq. (27)]. Thus we see that $\Delta Z_{V}^{\text {loc }}$ is compatible with being, as expected, purely a discretization effect.

We have shown here that the $Z_{V}$ value obtained for the nonconserved local HISQ current using the RI-SMOM scheme is indeed exact i.e., it has no nonperturbative condensate contributions (visible at our high level of accuracy) that would survive the continuum limit as a source of systematic error. This can be traced to the fact that the condensate contributions present in the vector vertex function for the conserved vector current and in the inverse propagator must cancel because of the Ward-Takahashi identity. This identity also protects $Z_{V}$ from any effects arising from the gauge-fixing procedure. 

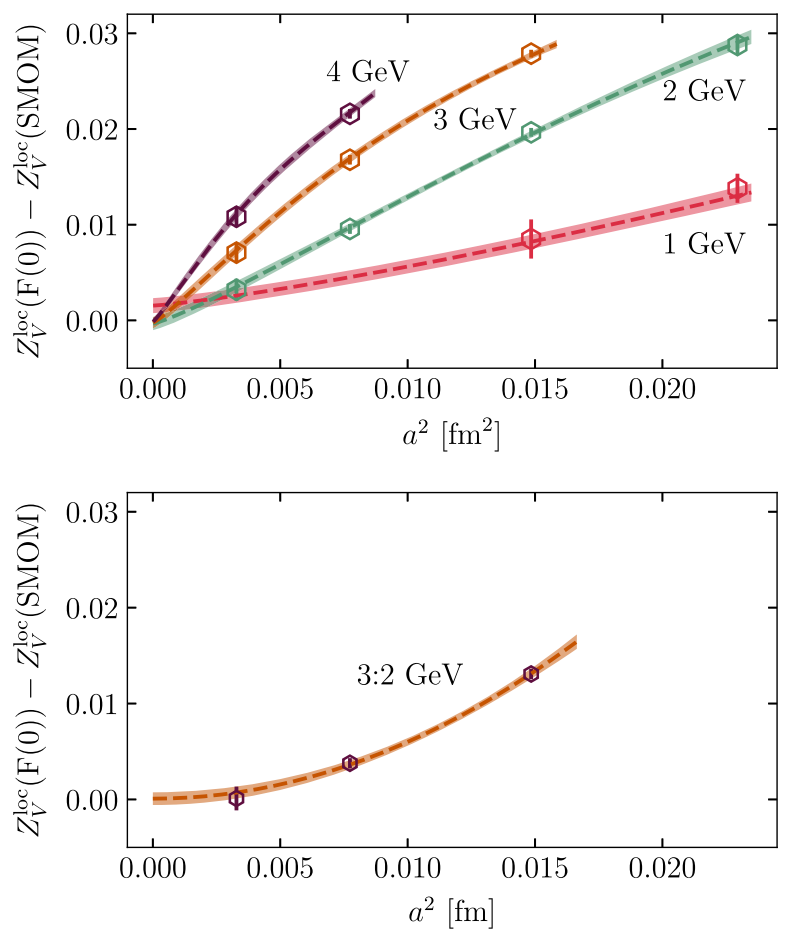

FIG. 6. The top plot shows $Z_{V}^{\text {loc }}(\mathrm{SMOM})$ for $\mu$ values between 1 and $4 \mathrm{GeV}$, plotted as a difference to the corresponding $Z_{V}$ at that lattice spacing obtained from the vector form factor at zero momentum transfer. The fit shown (see text) accounts for discretization errors and condensate contributions, but no condensate contributions are seen and they are strongly constrained to zero by the fit. The lower plot shows the same difference but for a $Z_{V}^{\text {loc }}(\mathrm{SMOM})$ derived from results at $\mu=2$ and $3 \mathrm{GeV}$ in such a way as to reduce discretization effects [see Eq. (30)]. The fit here is to a simple (constant $+a^{4}$ ) form as described in the text.

This means that there is in fact no lower limit in principle to the $\mu$ value that can be used for the vector current renormalization in the RI-SMOM scheme. In Fig. 6 (top plot) we include values corresponding to $\mu=1 \mathrm{GeV}$. These show smaller discretization effects than those for the higher $\mu$ values and so may be preferable on these grounds if only one $\mu$ value is used (which is all that is necessary in principle since no allowance needs to be made for condensate effects). The statistical errors possible with 20 configurations grow as $\mu$ is reduced. However, for $\mu=$ $1 \mathrm{GeV}$ the uncertainties could still readily be reduced to the $0.1 \%$ level with higher statistics.

Smaller discretization effects are possible by extrapolating in $\mu$ to $\mu=0$. A simple method that removes $\mu^{2} a^{2}$ terms in $Z_{V}^{\text {loc }}(\mathrm{SMOM})$ combines results at two different $\mu$ values (for a given lattice spacing) to determine a new value

$$
\begin{aligned}
& Z_{V}^{\text {loc }}(\operatorname{SMOM})\left(\mu_{1}, \mu_{2}\right) \\
& \quad=\frac{\mu_{1}^{2} Z_{V}^{\text {loc }}(\operatorname{SMOM})\left(\mu_{2}\right)-\mu_{2}^{2} Z_{V}^{\text {loc }}(\operatorname{SMOM})\left(\mu_{1}\right)}{\mu_{1}^{2}-\mu_{2}^{2}} .
\end{aligned}
$$

This can always be done, given that $Z_{V}^{\text {loc }}(\mathrm{SMOM})$ only depends on $\mu$ through discretization effects. We use $\mu_{1}=$ $3 \mathrm{GeV}$ and $\mu_{2}=2 \mathrm{GeV}$ and Eq. (30) returns a precise result because the statistical uncertainties are very small on these $\mu$ values. We show the results of taking a difference to $Z_{V}^{\text {loc }}(\mathrm{F}(0))$ for this new $Z_{V}$ value in the lower plot of Fig. 6 . The points clearly have smaller discretization effects compared to the original $Z_{V}$ values that they were derived from. Given that the discretization effects in $Z_{V}^{\text {loc }}(\mathrm{F}(0))$ were relatively small [11] we interpret this as a reduction of discretization effects in $Z_{V}^{\text {loc }}(\mathrm{SMOM})$. We can fit the points in the lower plot of Fig. 6 to a very simple curve, $C+D(a \times 1 \mathrm{GeV})^{4}$ and $C$ is found to be $0.00008(66)$. The smaller discretization effects seen using Eq. (30) may make this approach preferable to that of using $Z_{V}^{\text {loc }}(\mathrm{SMOM})$ for a single $\mu$ value although it doubles the cost. Using three values of $\mu$ a higher-order scheme could obviously be devised to reduce discretization effects further.

\section{E. $Z_{V}$ for the local current in the $R I^{\prime}$-MOM scheme}

We now turn to the determination of the renormalization constant for the nonconserved local vector current using the RI'-MOM scheme, $Z_{V}^{\text {loc }}(\mathrm{MOM})$. Again, the vector vertex function must be a taste singlet. The RI'-MOM scheme uses a simple $\gamma_{\mu}$ projector [Eq. (16)], which for the HISQ local vector current needs to have spin taste $\gamma_{\mu} \otimes \gamma_{\mu}$. Then we use

$$
\sum_{\mu} \overline{\overline{\left(\gamma_{\mu} \otimes \gamma_{\mu}\right)}} \Lambda_{V, \text { loc }}^{\mu}
$$

to determine $Z_{V} / Z_{q}$ along with Eq. (20) to determine $Z_{q}$. Figure 7 shows the valence mass extrapolation for one set of raw results. Despite having a more significant mass extrapolation than for the RI-SMOM results (Fig. 5), this is still very mild. Table III gives our results in column 4, where we note that the values given for $Z_{V}^{\text {loc }}(\mathrm{MOM})$ include

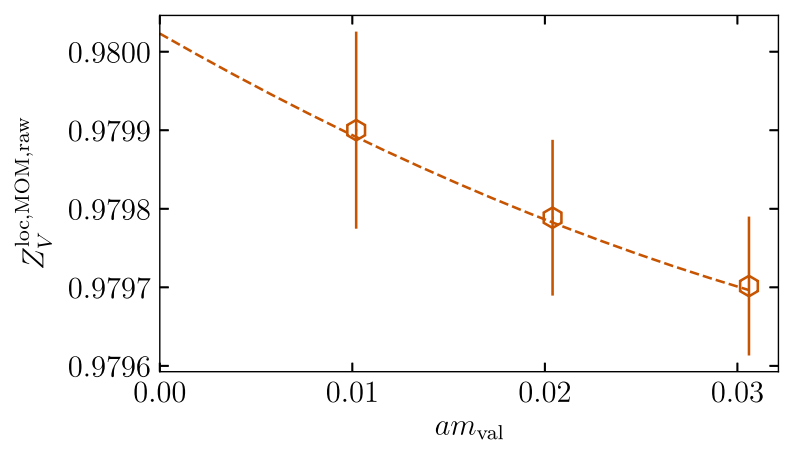

FIG. 7. Valence mass dependence of our raw results for $Z_{V}^{\text {loc }}$ calculated in the RI'-MOM scheme, before multiplication by the additional renormalization factor needed to match to $\overline{\mathrm{MS}}$. Results and extrapolation are shown for $\mu=3 \mathrm{GeV}$ on Set 2 . 


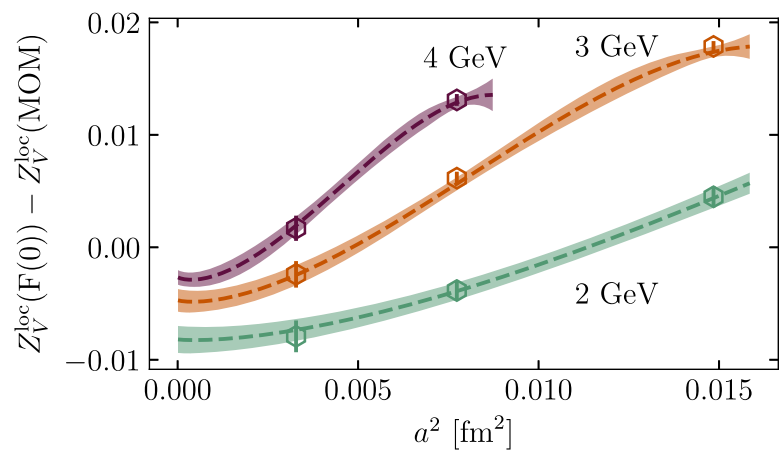

FIG. 8. $\quad Z_{V}^{\mathrm{loc}}(\mathrm{MOM})$ for $\mu$ values between 2 and $4 \mathrm{GeV}$, plotted as a difference to the corresponding $Z_{V}$ at that lattice spacing obtained from the vector form factor at zero momentum transfer. The fit shown (see text) accounts for discretization errors and condensate contributions, with condensate contributions being necessary to obtain a good fit.

the additional renormalization factor shown in Eq. (25) and given in Table II.

Figure 8 shows our results given, following the discussion in Sec. IV D, as a difference to the renormalization constants obtained for the local current using the form factor method in Ref. [11]. This figure is very different from Fig. 6, with the results showing no sign of converging to zero in the continuum limit that would demonstrate agreement between the form factor and $\mathrm{RI}^{\prime}-\mathrm{MOM}$ schemes for $Z_{V}$. This shows the presence of condensate contributions in $Z_{V}^{\text {loc }}(\mathrm{MOM})$ and to fit these results we need to include condensates that survive the continuum limit in the fit form.

For the difference of $Z_{V}^{\text {loc }}$ values shown in Fig. 8 we use the same fit form as that used earlier for the RI-SMOM results in Eq. (29) [with the addition of an $\alpha_{s}^{4}$ to allow for uncertainty in the matching from MOM to $\overline{\mathrm{MS}}$ as used in Eq. (26); this term has very little effect]. This fit, with $\chi^{2} /$ d.o.f. $=0.14$ is shown by the dashed lines in Fig. 8. It returns a coefficient for the leading-order condensate term of $-0.209(63)$ which is consistent with the leading-order condensate term seen in the conserved current $Z_{V}$ calculated in the RI'-MOM scheme [Eq. (27), with opposite sign because of our definition of $\Delta Z_{V}$ here]. Note the difference with the results in the RI-SMOM case.

The results of Fig. 8 show that the standard RI'-MOM scheme cannot be used to determine an accurate result for $Z_{V}$ for nonconserved currents. If no attention is paid to the contamination of $Z_{V}$ by condensate contributions then $\mathcal{O}(1 \%)$ systematic errors will be made.

We can modify the RI'-MOM scheme to address this issue, however. We know that the conserved current and the renormalized local current are the same operator in the continuum limit and so their vertex functions must contain the same nonperturbative contributions from the $\mathrm{RI}^{\prime}$-MOM scheme in that limit. We can therefore calculate $Z_{V}^{\text {loc }}(\mathrm{MOM})$ by taking a ratio of the vertex functions of the

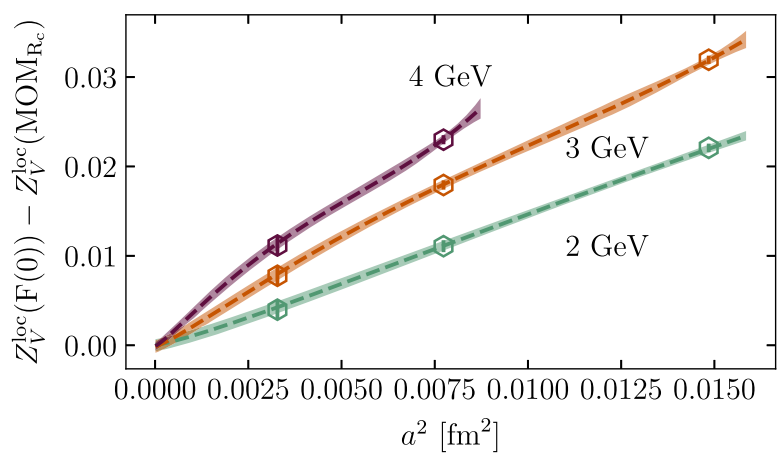

FIG. 9. $\quad Z_{V}^{\text {loc }}\left(\mathrm{MOM}_{\mathrm{Rc}}\right)$ from the modified RI'-MOM scheme of Eq. (32), plotted as a difference to the corresponding $Z_{V}$ at that lattice spacing obtained from the vector form factor at zero momentum transfer. Results are shown for $\mu$ values from 2 to $4 \mathrm{GeV}$. The fit shown (see text) accounts for discretization errors and condensate contributions, but condensate contributions are strongly constrained to be zero.

local and conserved currents. We call this scheme the $\mathrm{RI}^{\prime}-\mathrm{MOM}_{\mathrm{Rc}}$ scheme. Specifically we calculate

$$
Z_{V}^{\text {loc }}\left(\mathrm{MOM}_{\mathrm{Rc}}\right)=\frac{\operatorname{Tr}\left(\gamma_{\mu} \Lambda_{V, \text { cons }}^{\mu}\right)}{\operatorname{Tr}\left(\gamma_{\mu} \Lambda_{V, \text { loc }}^{\mu}\right)}=\frac{Z_{V}^{\text {loc }}(\mathrm{MOM})}{Z_{V}^{\text {cons }}(\mathrm{MOM})}
$$

Taking the ratio also means that no additional renormalization is needed in this case.

Our results from implementing this scheme are given in Table III (column 5). Figure 9 shows the results given once again as a difference to the renormalization constant obtained for the local current in the form factor method. We now see that the difference of $Z_{V}$ values clearly approaches 0 in the continuum limit and there is no sign of condensate contamination in that limit. The results in the $\mathrm{RI}^{\prime}-\mathrm{MOM}_{\mathrm{Rc}}$ scheme look very similar to those in the RI-SMOM scheme (see Fig. 6). We can fit the values for $\Delta Z_{V}^{\text {loc }}$ in Fig. 9 to the same form as that used for the RI-SMOM results [Eq. (29)]. The fit gives $\chi^{2} /$ d.o.f. $=0.32$ and constrains the lowest-order condensate coefficient that would survive the continuum limit to $-0.01(5)$.

We conclude that the modified RI'-MOM scheme of Eq. (32) does provide a method to determine an accurate renormalization for the local vector current. The method does require calculations with the conserved current and so is more complicated than the RI-SMOM scheme.

\section{F. $Z_{V}$ for the local current in the RI-SMOM $\gamma_{\mu}$ scheme}

An alternative momentum-subtraction scheme is the RI-SMOM $\gamma_{\mu}$ scheme which uses the same vertex function (and wave-function renormalization) as the RI'-MOM scheme but uses RI-SMOM kinematics (i.e., $q=p_{1}-$ $p_{2} \neq 0, p_{1}^{2}=p_{2}^{2}=q^{2}=\mu^{2}$. 


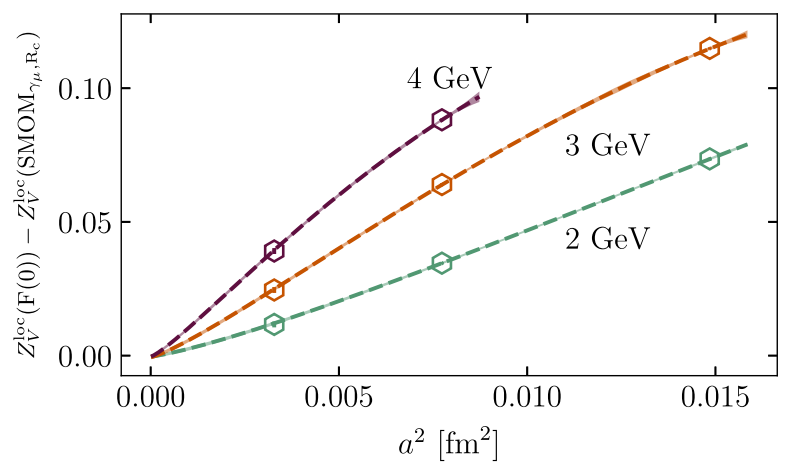

FIG. 10. $Z_{V}^{\text {loc }}\left(\mathrm{SMOM}_{\gamma_{\mu}, \mathrm{Rc}}\right)$ from the modified RI-SMOM $\gamma_{\mu}$ scheme, plotted as a difference to the corresponding $Z_{V}$ at that lattice spacing obtained from the vector form factor at zero momentum transfer. Results are shown for $\mu$ values from 2 to $4 \mathrm{GeV}$. The fit shown (see text) accounts for discretization errors and condensate contributions, but condensate contributions are strongly constrained to be zero.

To obtain an accurate result for $Z_{V}$ for the local current (as an example of a nonconserved current) we must modify the scheme as was done for the RI'-MOM scheme in Eq. (32). The only difference is that we must also modify the tree-level vertex function factor for the conserved current from that of Eq. (24) to reflect the SMOM kinematics. Table III gives our results from this modified RI-SMOM $\gamma_{\mu}, \mathrm{Rc}$ scheme in column 6. Figure 10 plots the difference of these $Z_{V}$ values with those from using the form factor method. We see that, as for the SMOM scheme in Fig. 6 and the modified RI'-MOM scheme in Fig. 9, the values converge to zero as $a \rightarrow 0$ as discretization effects should. Discretization effects are significantly larger here than in the previous schemes, however. We fit the results to the same functional form as used for the other schemes [i.e., Eq. (29)] and obtain a good fit [we double the prior width on $(a \mu)^{n}$ terms to allow for the larger discretization effects]. $\chi^{2} /$ d.o.f. $=0.32$ and the lowest-order condensate coefficient is constrained very tightly, as in the other exact cases, to $-0.03(5)$.

The same conclusions apply as for the RI'-MOM scheme, i.e., that defining $Z_{V}$ from the ratio of vertex functions with the conserved current gives an exact result.

\section{G. Renormalization of the axial-vector current}

The renormalization factors for axial-vector currents can also be calculated using momentum-subtraction schemes. However, for actions with sufficient chiral symmetry the axial-vector current renormalization, $Z_{A}$, can be related to the vector current renormalization at zero quark mass. For example, for staggered quarks, $Z_{S \otimes T}=Z_{S 5 \otimes T 5}$ to all orders in perturbation theory [34]. Here $S \otimes T$ indicates the operator spin taste and $S 5=\gamma_{5} S$. This means that the local axial vector current and local vector current have the same renormalization factor.

Having shown that the local vector current renormalization factor can be calculated accurately and without

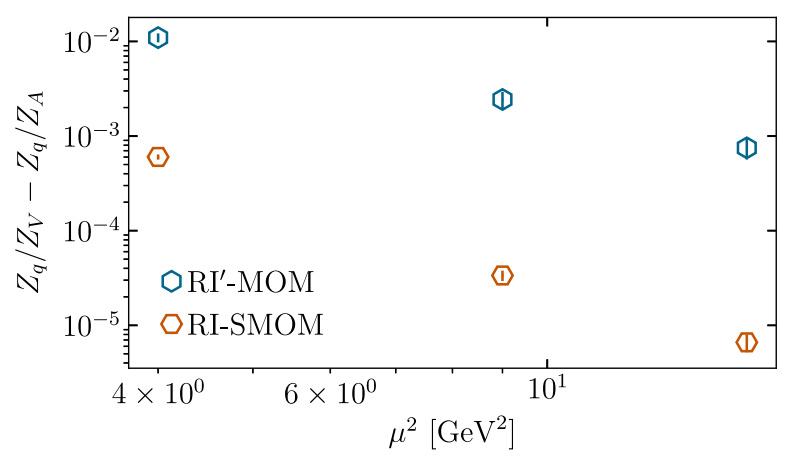

FIG. 11. The difference between the vertex functions for the local vector and local axial-vector currents as a function of $\mu$ in the RI'-MOM and RI-SMOM schemes. The RI-SMOM scheme gives a much smaller nonperturbative contribution to $Z_{A}-Z_{V}$ than the $\mathrm{RI}^{\prime}$-MOM scheme, but neither scheme gives $Z_{V}=Z_{A}$ which is true to all orders in perturbation theory. The values plotted result from an extrapolation to zero valence quark mass and are shown for the finest lattice we use (Set 7 in Table I).

contamination by condensate contributions in the RI-SMOM scheme in Sec. IV D, it therefore makes sense to also use this value for the local axial-vector current. Indeed, doing a separate calculation of $Z_{A}^{\text {loc }}$ risks introducing condensate contributions where none would be found using $Z_{A}=Z_{V}$. Figure 11 shows the difference between the local vector and local axial-vector vertex functions after extrapolation to zero quark mass, on the superfine lattices (Set 7). Each point plotted is the difference of the local vector and local axial-vector vertex functions i.e., $Z_{q} / Z_{V}-Z_{q} / Z_{A}$.

We see that the difference in the RI-SMOM scheme is small but not zero. The results demonstrate approximately $\mu^{-6}$ behavior as expected on the basis of a chiral-symmetrybreaking condensate contribution [14]. Note that this contribution comes from $Z_{q} / Z_{A}$. For the RI'-MOM scheme the difference is much larger than for RI-SMOM and has a smaller slope in this log-log plot. This reflects the known impact of chiral-symmetry-breaking nonperturbative artifacts in this scheme [14]. In both cases it would be preferable to use $Z_{A}=Z_{V}$, in the RI'-MOM case using the modified $\mathrm{RI}^{\prime}-\mathrm{MOM}_{\mathrm{Rc}}$ approach of Eq. (32).

\section{INCLUDING QUENCHED QED EFFECTS}

As lattice QCD calculations reach subpercent precision it will become necessary to evaluate the electromagnetic corrections expected at this level. If QED effects are included in calculations involving nonconserved vector currents, such as the ongoing Fermilab/HPQCD/MILC calculations of the hadronic vacuum polarization contribution to the anomalous magnetic moment of the muon [35], then consistency requires that QED effects are also included in the vector current renormalization. Here we will study the impact of the valence quarks having electric charge on the renormalization of the local vector current 
using the RI-SMOM scheme (for earlier results using different methods see Refs. [36,37]).

We include "quenched QED" in our lattice calculations by multiplying our QCD gauge fields by a U(1) gauge field representing the photon. The photon field, $A_{\mu}(k)$, is randomly generated in momentum space from a Gaussian distribution with variance $1 / \hat{k}^{2}$ to yield the correct $\mathcal{O}\left(a^{2}\right)$ improved Feynman gauge propagator on the lattice [the definition of $\hat{k}$ is given in Eq. (21)]. $A_{\mu}(k)$ is then converted to Landau gauge and transformed to position space. To ensure the correct gauge covariance in position space it is important to remember that the position of the gauge fields is at the center of the links, and not the sites [38]. The $A_{\mu}$ field in position space is then used as the phase to construct a $\mathrm{U}(1)$ field [39] in the form $\exp \left(i e Q A_{\mu}\right)$ where $Q$ is the charge of the quark that will interact with the field, in units of the charge on the proton, $e$. We use the $\mathrm{QED}_{L}$ formulation of compact QED [40], in which all zero modes are set to zero, $A_{\mu}\left(k_{0}, \mathbf{k}=0\right)=0$ with $A_{\mu}$ in Landau gauge (for a review of approaches to handling zero modes in QED on the lattice see Ref. [41]). We multiply the gluon field for each link of the lattice by the appropriate $\mathrm{U}(1)$ field before applying the HISQ smearing. The valence quarks can then interact with the photon via the standard HISQ action. Note that the sea quarks remain electrically neutral, so this is not a fully realistic scenario. Nevertheless it allows us to evaluate the most important QED effects.

We have tested that the $\mathrm{U}(1)$ configurations we generate correctly reproduce the $\mathcal{O}\left(\alpha_{\mathrm{QED}}\right)$ perturbation theory prediction for the average plaquette [42], independent of gauge choice. Our results for the average value of the U(1) link field also agree with the $\mathcal{O}\left(\alpha_{\mathrm{QED}}\right)$ expectations:

$$
\begin{gathered}
\text { Landau gauge: } 1-\alpha_{\mathrm{QED}} Q^{2} 0.0581, \\
\text { Feynman gauge: } 1-\alpha_{\mathrm{QED}} Q^{2} 0.0775 .
\end{gathered}
$$

Note that the Landau gauge $\mathcal{O}\left(\alpha_{\mathrm{QED}} Q^{2}\right)$ coefficient is $1 / C_{F}=3 / 4$ that of the corresponding QCD result for the $a^{2}$-improved gluon action [43] since the gluon propagator then has the same form as that of the photon here. The Feynman gauge coefficient is then $4 / 3$ of the Landau gauge coefficient.

Although we have tested calculations as a function of quark charge, $Q$, the results we will show here are all for $Q=2 / 3$. The results are not extrapolated to zero valence quark mass and are instead just the values at the sea light quark mass on each ensemble. The valence mass dependence of the results is observed to be negligibly small, as was the case in pure QCD.

An important test of the interaction between the quarks and the QCD + QED gauge fields is that $Z_{V}=1$ for the QCD + QED conserved current in the RI-SMOM scheme, as expected from a trivial extension of the Ward-Takahashi identity to this case. This is demonstrated in Fig. 12.

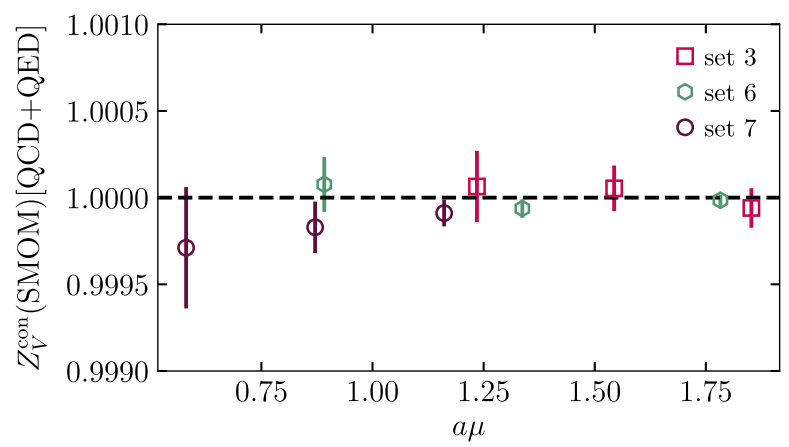

FIG. 12. Results for the renormalization factor, $Z_{V}$ for the QCD + QED conserved current for the HISQ action, calculated using the RI-SMOM scheme. Results are given for coarse, fine and superfine gluon-field configurations for quark electric charge, $Q=2 e / 3$ and a variety of momenta with magnitude $a \mu$ in lattice units.

Our analysis for the renormalization of the local vector current in the RI-SMOM scheme will include the ratio of the $Z_{V}^{\text {loc }}$ calculated with and without the inclusion of electromagnetic effects. We proceed exactly as for the pure QCD case discussed in Sec. IV D. The strong correlations between the QCD and QCD + QED calculations allow a very precise determination of this ratio (a typical correlation being $\sim 0.99$ ). We will denote a quantity $X$ calculated in pure $\mathrm{QCD}$ as $X[\mathrm{QCD}]$ while the same quantity calculated with the inclusion of QED effects will be denoted as $X[\mathrm{QED}+$ $\mathrm{QCD}]$. We will also employ the notation $X[(\mathrm{QCD}+\mathrm{QED}) /$ $\mathrm{QCD}] \equiv X[\mathrm{QED}+\mathrm{QCD}] / X[\mathrm{QCD}]$.

Because QED is a long-range interaction it is important to test finite-volume effects, although we do not expect them to be large here since we are studying the renormalization of electrically neutral currents. The finite-volume effects in the self-energy function of fermions has been studied in Ref. [44] with the result that for off-shell quarks the finite-volume effects start at order $1 / L_{s}^{2}$ where $L_{s}$ is the lattice spatial extent. This implies that even the finite-volume effects for quantities such as $Z_{q}$ should be small. Figure 13 confirms both of these expectations with results on the three lattice sets with the same parameters but different volumes (Sets 3, 4 and 5, ranging in spatial extent from 2.9 to $4.9 \mathrm{fm}$ ). Negligible effects are seen here and we therefore ignore finite-volume issues in the following analysis.

Our results for the effect of quenched QED on $Z_{V}$ for the local HISQ current in the RI-SMOM scheme are given for $\mu$ values from 2 to $4 \mathrm{GeV}$ and at three values of the lattice spacing in Table IV. The results are plotted in Fig. 14.

Given our results for the pure QCD case in Sec. IV D we expect the results for $Z_{V}$ for QCD + QED to be similarly well behaved. We therefore perform a fit to the ratio of $Z_{V}$ for QCD + QED to that for pure QCD results that allows for both discretization effects along with a perturbative expansion for the ratio of renormalization constants. The leading QCD effects will cancel between the numerator and 

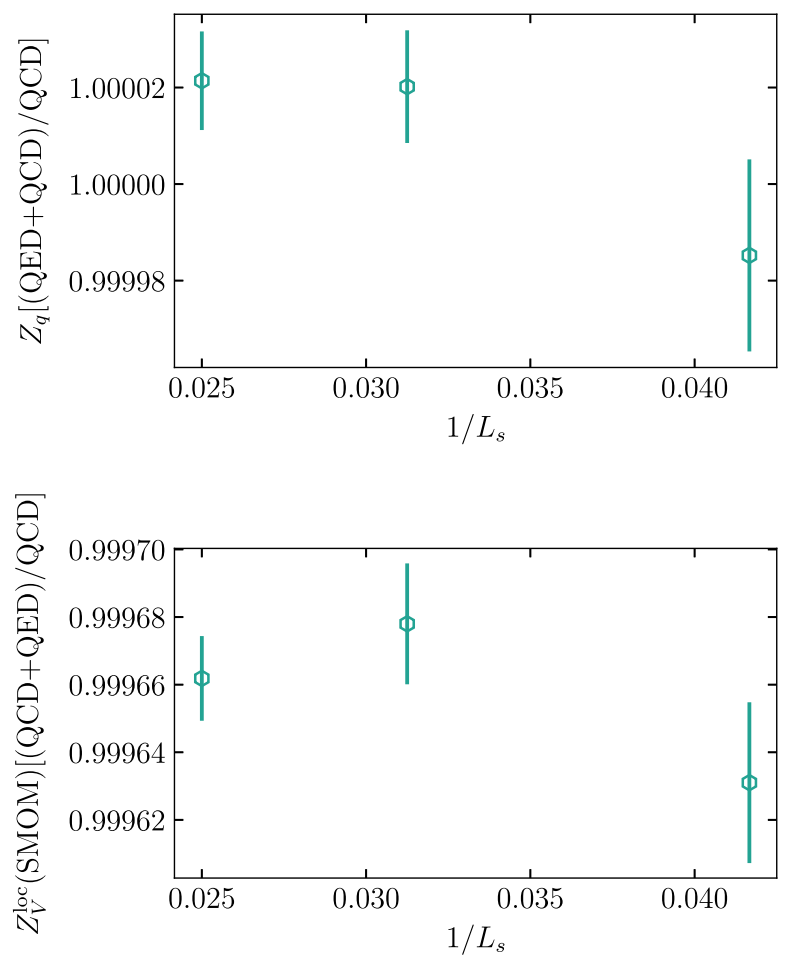

FIG. 13. The impact of quenched QED (with quark charge $2 e / 3$ ) on the determination of $Z_{V}^{\text {loc }}$ and $Z_{q}$ using the RI-SMOM scheme as a function of the lattice spatial extent, $L_{s}$ in lattice units. Results are for coarse lattices (Sets 3,4 and 5), and $\mu=2 \mathrm{GeV}$. The volume dependence is negligible.

denominator of the ratio and so the leading term in this expansion will be $\mathcal{O}\left(\alpha_{\mathrm{QED}}\right)$. We can even fix the coefficient of the leading-order term based on the QCD perturbation theory for the pure QCD case. The $\mathcal{O}\left(\alpha_{s}\right)$ coefficient for $Z_{V}^{\text {loc }}$ for pure QCD is $-0.1164(3)$ [11]. We therefore expect that the coefficient of $\alpha_{\mathrm{QED}} Q^{2}$ in the QED case is $-0.1164 \times 3 / 4=-0.0873$. For $Q=2 e / 3$ this corresponds to an $\mathcal{O}\left(\alpha_{\mathrm{QED}}\right)$ coefficient of -0.0388 . This gives a leading-order result for $Z_{V}^{\text {loc }}$ of 0.9997 , which is very close to 1 . There will be in principle $\alpha_{s} \alpha_{\mathrm{QED}}$ corrections to this which are likely to have an even smaller impact.

We therefore take a fit form for the ratio of $Z_{V}$ values given in Table IV of

$$
\begin{aligned}
& Z_{V}^{\mathrm{loc}}(\mathrm{SMOM})[(\mathrm{QED}+\mathrm{QCD}) / \mathrm{QCD}] \\
& \quad=1+\alpha_{\mathrm{QED}}\left(\sum_{i} c_{i} \alpha_{s}^{i}\left(1+\sum_{j} d_{i j}(a \mu)^{2 j}\right)\right) .
\end{aligned}
$$

We use $i=0,1,2,3$ and $j=1,2,3$ fixing $c_{0}$ to the value given above. Note that $\alpha_{\mathrm{QED}}$ does not run in this expression because we are using quenched QED. $\alpha_{s}$ in Eq. (34) is taken as $\alpha_{\overline{\mathrm{MS}}}(1 / a)$. This fit returns a $\chi^{2} /$ d.o.f. value of 0.25. The fit is plotted with the results in Fig. 14 .

Figure 14 shows that the results for $Z_{V}$ behave as expected. The impact of quenched QED on the value of
TABLE IV. The ratio of renormalization factors $Z_{V}$ for the $\mathrm{QCD}+$ quenched QED case to the pure QCD case. These are for the local HISQ vector current calculated in the RI-SMOM scheme on gluon-field configuration sets listed in column 1 and at $\mu$ values listed in column 2 (and at a valence quark mass of $m_{l}$ ).

\begin{tabular}{lcc}
\hline \hline Set & $\mu[\mathrm{GeV}]$ & $Z_{V}^{\text {loc }}(\mathrm{SMOM})[(\mathrm{QED}+\mathrm{QCD}) / \mathrm{QCD}]$ \\
\hline 3 & 2 & $0.999631(24)$ \\
6 & 2 & $0.999756(32)$ \\
7 & 2 & $0.999831(43)$ \\
3 & 2.5 & $0.999615(12)$ \\
3 & 3 & $0.999622(13)$ \\
6 & 3 & $0.9997043(39)$ \\
7 & 3 & $0.9997797(92)$ \\
6 & 4 & $0.9996754(26)$ \\
7 & 4 & $0.9997425(24)$ \\
\hline \hline
\end{tabular}

$Z_{V}^{\text {loc }}$ is tiny and indeed negligible if we imagine working to an accuracy of $0.1 \%$. Note that this follows directly from the analysis above in which we derived the $\mathcal{O}\left(\alpha_{\mathrm{QED}}\right)$ coefficient for the QCD + QED case from the pure QCD case. Because the HISQ action is so highly improved $Z_{V}^{\text {loc }}$ is very close to 1 in the pure $\mathrm{QCD}$ case. It then has to be true that the difference from 1 in $Z_{V}$ induced by QED will be over 100 times smaller than that induced by QCD. For the HISQ action this means that the impact of QED in $Z_{V}^{\text {loc }}$ is of order $0.03 \%$. This should be contrasted with the case from the domain-wall action where the $Z_{V}$ value in pure QCD is 0.7 and so the impact of quenched QED is to change $Z_{V}$ by approximately $0.3 / 100$ for $Q=2 e / 3$, which in this case is $0.3 \%$ (see Table 6 of Ref. [36]); this is not negligible.

The effect of having electrically charged sea quarks would appear in $Z_{V}$ at $\mathcal{O}\left(\alpha_{s}^{2} \alpha_{\mathrm{QED}}\right)$ i.e., two orders in $\alpha_{s}$

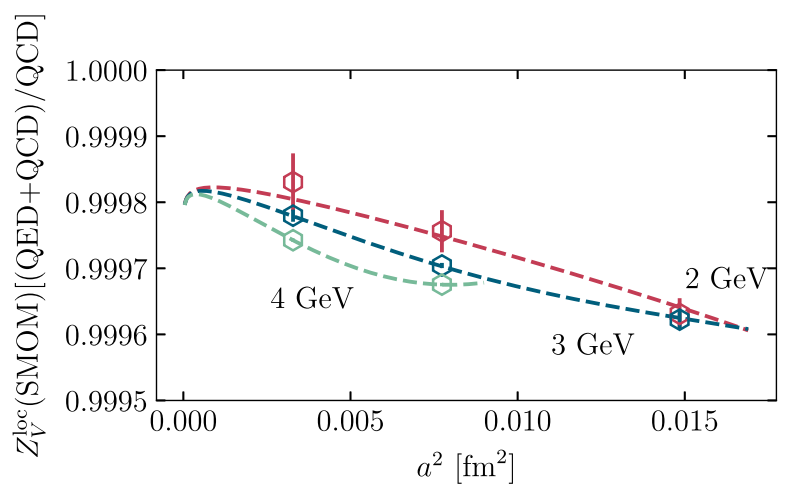

FIG. 14. The ratio of $Z_{V}^{\text {loc }}$ values for QCD + QED to QCD calculated in the RI-SMOM scheme. Results are given for coarse to superfine lattices at $\mu$ values from 2 to $4 \mathrm{GeV}$ and plotted against the square of the lattice spacing. The dashed lines give the result of a fit described in the text that shows that the results are fully described by a perturbative series (of which the leading coefficient is known) up to discretization effects. The dips in the fit functions close to $a=0$ are the result of the fact that the argument of $\alpha_{s}$ in the fit function [Eq. (34)] is inversely related to $a$. 
below the leading term; the leading term comes from a photon exchange across a quark bubble created from a gluon. This is unlikely to change the picture significantly.

The effect of QED on $Z_{V}$ is of course not a physical result and it needs to be combined with hadronic matrix elements for the vector current to understand the physical effect of QED. For this we simply take the values for $Z_{V}$ at a fixed $\mu$ value for the ensembles for which we have matrix element results, multiply them and extrapolate to the continuum limit. Different quark formalisms should agree on the physical effect (on an uncharged sea). We will give an analysis of the impact of quenched QED on vector current matrix elements calculated with the HISQ action elsewhere.

\section{CONCLUSIONS}

We have shown by explicit calculation how the vector Ward-Takahashi identity works for the HISQ action in lattice QCD. Renormalization methods that make use of this identity will give a renormalization constant of 1 for the conserved current as would be obtained in continuum QCD. The RI-SMOM momentum-subtraction scheme is such a scheme but the RI'-MOM scheme is not and this has implications for the accuracy achievable for $Z_{V}$ for nonconserved currents within each scheme. Our calculations have used the HISQ action but our conclusions are not specific to this action.

The RI-SMOM scheme provides precise values for $Z_{V}$ for nonconserved currents (using momentum sources) that are completely nonperturbative. Our results show that the $Z_{V}$ values are "exact" in being free of condensate contamination. This means that we can simply determine $Z_{V}$ at a given momentum scale $\mu$ on a given gluon-field ensemble, multiply our vector current hadronic matrix element by it and then extrapolate results for the renormalized matrix element to the continuum limit. Because there is no condensate contamination there is no lower limit to the $\mu$ value that can be used. Statistical errors grow as $\mu$ is reduced but discretization effects become smaller. In Sec. IV D we demonstrated a simple method to reduce discretization effects, if they are an issue, by combining results from two different $\mu$ values.

The $\mathrm{RI}^{\prime}$-MOM scheme can also provide precise values for $Z_{V}$ for nonconserved currents, but it is not completely nonperturbative. A more critical problem with this scheme is that the $Z_{V}$ values for both conserved and nonconserved currents have condensate contributions that begin at $1 / \mu^{2}$.
This means that the $Z_{V}$ values cannot be used to obtain accurate renormalized vector current matrix elements in the continuum limit without an analysis of these condensate contributions. This requires numbers for $Z_{V}$ at multiple $\mu$ values and a fit that includes condensate terms. If this analysis is not done, the results obtained in the continuum limit will be incorrect at the $1 \%$ level.

An alternative to the standard $\mathrm{RI}^{\prime}-\mathrm{MOM}$ scheme that avoids this problem is to determine $Z_{V}$ from a ratio of vector vertex functions for the conserved and nonconserved currents. We call this scheme $\mathrm{RI}^{\prime}-\mathrm{MOM}_{\mathrm{Rc}}$. A similarly modified RI-SMOM $\gamma_{\mu}$ scheme can also be used to obtain an exact $Z_{V}$. These schemes were discussed in Secs. IV E and IV F.

It is straightforward to include quenched QED effects in the determination of the vector current renormalization factor in a fully nonperturbative way using the RI-SMOM scheme and to obtain a full understanding of the results (including consistency with perturbation theory). We see only very small (below $0.1 \%$ ) effects for the local HISQ vector current reflecting the fact that the renormalization factors in the pure QCD case are already very close to 1 . We will include the QCD + QED $Z_{V}$ values in a future QCD + QED determination of hadronic vector current matrix elements.

\section{ACKNOWLEDGMENTS}

We are grateful to the MILC Collaboration for the use of their configurations and their code base. We thank E. Follana and E. Royo-Amondarain for gauge-fixing the superfine and fine configurations and we are grateful to E. Follana, S. Sharpe and A. Vladikas for useful discussions. Computing was done on the Darwin supercomputer at the University of Cambridge High Performance Computing Service as part of the DiRAC facility, jointly funded by the Science and Technology Facilities Council, the Large Facilities Capital Fund of BIS and the Universities of Cambridge and Glasgow. We are grateful to the Darwin support staff for assistance. Funding for this work came from the Science and Technology Facilities Council and the National Science Foundation.

\section{APPENDIX A: HISQ CONSERVED CURRENT}

The forward HISQ conserved current corresponding to the simple backward finite difference operator $\Delta^{\mu,-}$ in Eq. (4) is given by

$$
\begin{aligned}
J^{\mu,+}(\tilde{x})= & \frac{1}{2}\left[\bar{\psi}(x) \gamma_{\mu} W_{\mu}(x) \psi(x+\hat{\mu})+\text { H.c. }\right]+\frac{1}{16}\left[\bar{\psi}(x) \gamma_{\mu} X_{\mu}(x) \psi(x+\hat{\mu})+\text { H.c. }\right] \\
& -\frac{1}{48}\left[\bar{\psi}(x-2 \hat{\mu}) \gamma_{\mu} X_{\mu}(x-2 \hat{\mu}) X_{\mu}(x-\hat{\mu}) X_{\mu}(x) \psi(x+\hat{\mu})\right. \\
& +\bar{\psi}(x-\hat{\mu}) \gamma_{\mu} X_{\mu}(x-\hat{\mu}) X_{\mu}(x) X_{\mu}(x+\hat{\mu}) \psi(x+2 \hat{\mu}) \\
& \left.+\bar{\psi}(x) \gamma_{\mu} X_{\mu}(x) X_{\mu}(x+\hat{\mu}) X_{\mu}(x+2 \hat{\mu}) \psi(x+3 \hat{\mu})+\text { H.c. }\right]
\end{aligned}
$$


where $W$ are HISQ links and $X$ are the links after the first level of HISQ smearing in the notation of Ref. [4]. Note that $J^{\mu,+}$ sits on the link between $x$ and $x+\hat{\mu}$; $\tilde{x}$ is the halfway point on that link. The backward conserved current $J^{\mu,-}$ is the same but with $x \rightarrow x-\hat{\mu}$ and $x+\hat{\mu} \rightarrow x$. More complicated conserved currents can be defined in conjunction with higher-order difference operators for $\Delta^{\mu, \pm}$ but we do not do that here.

\section{APPENDIX B: RENORMALIZATION OF THE ONE-LINK VECTOR CURRENT}

Quark-line disconnected contributions for vector current-current correlators require the use of a taste-singlet vector current for staggered quarks. This has the same taste as the conserved current but it is often more convenient to use a simpler current than the conserved one. Here we discuss the renormalization of the nonconserved one-link point-split vector current using momentum-subtraction schemes. The qualitative picture is the same as that for the local current and so we simply include RI-SMOM results in this Appendix for completeness. They are relevant to our ongoing calculations of, for example, the quark-line disconnected pieces of the hadronic vacuum polarization contribution to the anomalous magnetic moment of the muon.

We consider the one-link point-split vector current with spin taste $\left(\gamma_{\mu} \otimes I\right)$. The operators that we use include gluon fields between the point-split quark fields to maintain gauge invariance. We take these gluon fields to be "thin links" i.e., no smearing is applied to them. We considered the two simplest constructions of this current. One, which we denote the forward one-link operator, is the conserved current with all three-link terms removed:

$$
j_{\mu}^{1 \text { link }} \equiv \frac{1}{2 u_{0}} \bar{\psi}(x) \overline{\left(\gamma_{\mu} \otimes 1\right)} U_{\mu}(x) \psi(x+\hat{\mu})+\text { H.c. }
$$

The other one-link operator we consider is the symmetric operator

$$
\begin{aligned}
j_{\mu}^{1 \text { link-symm }} \equiv & \frac{1}{2 u_{0}} \bar{\psi}(x) \overline{\left(\gamma_{\mu} \otimes 1\right)} U_{\mu}(x) \psi(x+\hat{\mu}) \\
& +\frac{1}{2 u_{0}} \bar{\psi}(x) \overline{\left(\gamma_{\mu} \otimes 1\right)} U_{\mu}^{\dagger}(x-\hat{\mu}) \psi(x-\hat{\mu}) .
\end{aligned}
$$

The two definitions coincide with the MOM kinematics. In the SMOM case, while the values produced from the two different definitions are not identical they agree within our statistical uncertainties. In what follows we then only present results for the forward one-link current.

Note that in the definitions of the one-link current above we include a factor $1 / u_{0} . u_{0}$ is a "tadpole-improvement" factor [45] which can be used, as here, to reduce the mismatch between lattice currents containing gluon fields
TABLE V. Column 2 gives the tadpole-improvement factor $u_{0}$ used in the definition of the one-link current [Eq. (B1)]. This is the mean value of the gluon field $U_{\mu}$ in Landau gauge. Column 3 gives the results for the $Z_{V}$ values determined from the form factor using the matrix element of the temporal one-link current between two pions at rest. ${ }^{\text {a }}$ The asterisk next to Set 6 is to denote that the results given here are actually for another fine ensemble with $a m_{l}=0.0074, a m_{s}=0.037$ and $a m_{c}=0.44$.

\begin{tabular}{lll}
\hline \hline Set & \multicolumn{1}{c}{$u_{0}$} & $Z_{V}^{1 \operatorname{link}}(\mathrm{F}(0))$ \\
\hline 1 & $0.820192(14)$ & $1.0332(23)$ \\
2 & $0.834613(14)$ & $1.0307(7)$ \\
$6^{*}$ & $0.852477(9)$ & $1.0193(9)$ \\
7 & $0.870935(5)$ & $1.0064(28)$ \\
\hline \hline
\end{tabular}

${ }^{a}$ We thank J. Simone for providing the $u_{0}$ values and J. Koponen and A. C. Zimermmane-Santos for providing the $Z_{V}(\mathrm{~F}(0))$ values.

and their continuum counterparts. $u_{0}$ works by canceling universal effects from tadpole diagrams that arise from the construction of the lattice gluon field. $u_{0}$ can in principle be any suitable ensemble average of a function of the gluon field that achieves this. Here we use the mean value of the gluon field $U_{\mu}$ in Landau gauge as the most appropriate form of $u_{0}$ in this case. The values for $u_{0}$ depend on the ensemble and are listed in Table V. ${ }^{2}$

We proceed for the one-link case in the same way as for the cases discussed in the main body of the paper. The wavefunction renormalization is exactly the same as before. We calculate the vertex function for the one-link current using an appropriate projector. For the RI-SMOM case we use

$$
\left.\frac{Z_{q}(q)}{Z_{V}(q)}=\frac{i}{48 V_{\gamma \otimes I}^{1 \text { link }}} \sum_{\mu, \nu}\left(-i a \hat{q}_{\mu}\right) \frac{a \hat{q}_{\nu}}{(a \hat{q})^{2}} \operatorname{Tr} \overline{\overline{\left(\gamma_{\nu} \otimes I\right)}} \Lambda_{V}^{\mu}\right] .
$$

In determining $Z_{V}^{1 \text { link }}$ an additional technical detail for point-split operators is that we must divide the vertex function in the full theory by the result of the tree-level (noninteracting) case. This was discussed previously for the conserved current in the RI'-MOM case in Sec. IV C [and denoted $V_{\gamma \otimes I}$ in Eq. (23) and above]. The tree-level result for the forward one-link current for the RI-SMOM kinematics is

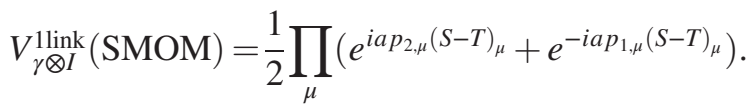

A further technical detail arises when using twisted boundary conditions to insert momentum with point-split operators in the vertex functions that we calculate. The propagator with twisted momentum can be written in terms of the untwisted one as

\footnotetext{
${ }^{2} Z_{V}$ for the tadpole-improved current is $u_{0}$ times $Z_{V}$ for the current with no tadpole improvement.
} 
TABLE VI. Renormalization factors for the (forward) one-link HISQ vector current for a variety of $\mu$ values (given in column 2) on gluon-field configurations at different lattice spacing values (denoted by the Set number in column 1). Column 3 gives results using the RI-SMOM scheme.

\begin{tabular}{lcc}
\hline \hline Set & $\mu[\mathrm{GeV}]$ & $Z_{V}^{1 \text { link }}(\mathrm{SMOM})$ \\
\hline 1 & 1 & $0.9617(11)$ \\
2 & 1 & $0.9713(19)$ \\
1 & 2 & $0.93516(16)$ \\
2 & 2 & $0.94966(20)$ \\
6 & 2 & $0.96695(11)$ \\
7 & 2 & $0.97996(34)$ \\
2 & 2.5 & $0.94236(11)$ \\
2 & 3 & $0.939193(87)$ \\
6 & 3 & $0.954643(37)$ \\
7 & 3 & $0.97225(12)$ \\
6 & 4 & $0.948641(20)$ \\
7 & 4 & $0.965353(56)$ \\
\hline \hline
\end{tabular}

$$
\tilde{S}(x, p)=e^{-i \theta x} S(x, p+\theta) .
$$

We want the vertex function for a point-split operator to take the following form (using a one-link operator $\Gamma$ as an example, but dropping the gluon fields for clarity):

$$
\begin{aligned}
& \sum_{x} \gamma_{5} e^{i\left(p_{1}+\theta_{1}\right) x} S^{\dagger}\left(x, p_{1}+\theta_{1}\right) \\
& \times \gamma_{5} \Gamma_{\mu} e^{-i\left(p_{2}+\theta_{2}\right) x} S\left(x+\hat{\mu}, p_{2}+\theta_{2}\right) \\
& =\sum_{x} \gamma_{5} e^{i p_{1} x} \tilde{S}^{\dagger}\left(x, p_{1}\right) \gamma_{5} \Gamma_{\mu} e^{-i p_{2} x} e^{i a \theta_{2, \mu}} \tilde{S}\left(x+\hat{\mu}, p_{2}\right) .
\end{aligned}
$$

The factor $e^{i a \theta_{2, \mu}}$ has to be inserted by hand.

Our results for $Z_{V}^{1 \text { link }}(\mathrm{SMOM})$ using the RI-SMOM scheme are given in Table VI for a variety of $\mu$ values for three values of the lattice spacing. We expect the $Z_{V}$ values obtained with RI-SMOM to be well behaved and free of condensate contributions because of the protection of the Ward-Takahashi identity, as for the local current discussed in Sec. IV D. We can test this, as was done for the local case, by taking a difference of the $Z_{V}$ values with those obtained from the form factor method.

The results for $Z_{V}$ from the form factor method are given in Table $\mathrm{V}$ for a variety of $\mu$ values and on ensembles with a range of lattice spacing values. The results for the

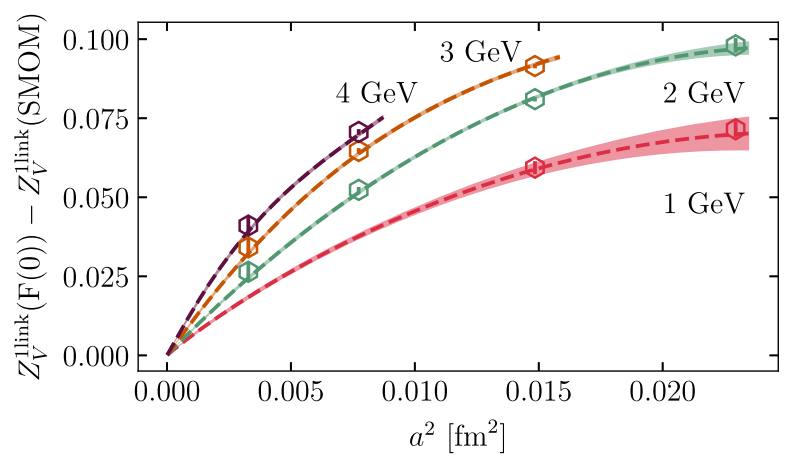

FIG. 15. $Z_{V}^{1 \text { link }}(\mathrm{SMOM})$ for $\mu$ values between 1 and $4 \mathrm{GeV}$, plotted as a difference to the corresponding $Z_{V}$ at that lattice spacing obtained from the vector form factor at zero momentum transfer. The fit shown [see Eq. (B7)] accounts for discretization effects only.

difference of $Z_{V}$ values between the form factor and RISMOM methods is plotted in Fig. 15. We show the results of a simple fit to a sum of possible discretization effects:

$$
\begin{aligned}
\Delta Z_{V}^{1 \operatorname{link}}(a, \mu)= & \sum_{i=0, j=1}^{2,3} c_{i j} \alpha_{s}^{i}(a \mu / \pi)^{2 j} \\
& +\sum_{i=0, j=1}^{2,3} d_{i j} \alpha_{s}^{i}(a \Lambda / \pi)^{2 j} .
\end{aligned}
$$

Here $\alpha_{s}$ is in the $\overline{\mathrm{MS}}$ scheme at a scale of $1 / a$. We have to include $(a \Lambda)^{n}$ terms as well as $(a \mu)^{n}$ terms here because of the relatively large discretization effects in the $Z_{V}$ values obtained from the form factor method. The priors on the coefficients of the fit are taken as $0 \pm 3$. The fit gives a $\chi^{2} /$ d.o.f. $=0.9$. This confirms that, again in this case, the RI-SMOM method gives a well-behaved result for $Z_{V}$.

The conclusion from this is that the renormalization factors for the one-link current obtained in the RI-SMOM scheme on the lattice can be used straightforwardly, and in a fully nonperturbative way, to renormalize matrix elements of the one-link current obtained in a lattice calculation. This means that values can be taken, e.g., from Table VI, for a fixed $\mu$ value on each ensemble. The $\mu$ chosen can take any value and the only limitation on taking it to have a small value (for minimal discretization effects) is that of the statistical errors that grow as $\mu$ is reduced.
[1] Q. Mason, H. D. Trottier, R. Horgan, C. T. H. Davies, and G. P. Lepage (HPQCD Collaboration), High-precision determination of the light-quark masses from realistic lattice QCD, Phys. Rev. D 73, 114501 (2006).
[2] A. T. Lytle, C. T. H. Davies, D. Hatton, G. P. Lepage, and C. Sturm (HPQCD Collaboration), Determination of quark masses from $n_{f}=4$ lattice QCD and the RI-SMOM intermediate scheme, Phys. Rev. D 98, 014513 (2018). 
[3] S. Naik, On-shell improved Lattice action for QCD with Susskind Fermions and asymptotic freedom scale, Nucl. Phys. B316, 238 (1989).

[4] E. Follana, Q. Mason, C. Davies, K. Hornbostel, G. P. Lepage, J. Shigemitsu, H. Trottier, and K. Wong (HPQCD and UKQCD Collaborations), Highly improved staggered quarks on the lattice, with applications to charm physics, Phys. Rev. D 75, 054502 (2007).

[5] E. Follana, C. T. H. Davies, G. P. Lepage, and J. Shigemitsu (HPQCD and UKQCD Collaboration), High Precision Determination of the pi, K, D and D(s) Decay Constants from Lattice QCD, Phys. Rev. Lett. 100, 062002 (2008).

[6] G. C. Donald, C. T. H. Davies, R. J. Dowdall, E. Follana, K. Hornbostel, J. Koponen, G. P. Lepage, and C. McNeile (HPQCD Collaboration), Precision tests of the $J / \psi$ from full lattice QCD: Mass, leptonic width and radiative decay rate to $\eta_{c}$, Phys. Rev. D 86, 094501 (2012).

[7] G. C. Donald, C. T. H. Davies, J. Koponen, and G. P. Lepage (HPQCD Collaboration), $V_{c s}$ from $D_{s} \rightarrow \phi \ell \nu$ semileptonic decay and full lattice QCD, Phys. Rev. D 90, 074506 (2014).

[8] G. C. Donald, C. T. H. Davies, J. Koponen, and G. P. Lepage (HPQCD Collaboration), Prediction of the $D_{s}^{*}$ Width from a Calculation of its Radiative Decay in Full Lattice QCD, Phys. Rev. Lett. 112, 212002 (2014).

[9] V. A. Novikov, M. A. Shifman, A. I. Vainshtein, and V. I. Zakharov, Wilson's operator expansion: Can it fail?, Yad. Fiz. 41, 1063 (1985) [Nucl. Phys. B249, 445 (1985)].

[10] A. Vladikas, Three topics in renormalization and improvement, in Modern Perspectives in Lattice QCD: Quantum Field Theory and High Performance Computing. Proceedings, International School, 93rd Session, Les Houches, France, 2009 (2011), pp. 161-222 [arXiv:1103.1323].

[11] B. Chakraborty, C. T. H. Davies, G. C. Donald, J. Koponen, and G. P. Lepage (HPQCD Collaboration), Nonperturbative comparison of clover and highly improved staggered quarks in lattice QCD and the properties of the $\phi$ meson, Phys. Rev. D 96, 074502 (2017).

[12] G. Martinelli, C. Pittori, C. T. Sachrajda, M. Testa, and A. Vladikas, A general method for non-perturbative renormalization of lattice operators, Nucl. Phys. B445, 81 (1995).

[13] K. G. Chetyrkin and A. Retey, Renormalization and running of quark mass and field in the regularization invariant and MS-bar schemes at three loops and four loops, Nucl. Phys. B583, 3 (2000).

[14] Y. Aoki et al., Non-perturbative renormalization of quark bilinear operators and $\mathrm{B}(\mathrm{K})$ using domain wall fermions, Phys. Rev. D 78, 054510 (2008).

[15] C. Sturm, Y. Aoki, N. H. Christ, T. Izubuchi, C. T. C. Sachrajda, and A. Soni, Renormalization of quark bilinear operators in a momentum-subtraction scheme with a nonexceptional subtraction point, Phys. Rev. D 80, 014501 (2009).

[16] M. Gockeler, R. Horsley, H. Oelrich, H. Perlt, D. Petters, P. E. L. Rakow, A. Schafer, G. Schierholz, and A. Schiller, Nonperturbative renormalization of composite operators in lattice QCD, Nucl. Phys. B544, 699 (1999).

[17] D. Becirevic, V. Gimenez, V. Lubicz, G. Martinelli, M. Papinutto, and J. Reyes, Renormalization constants of quark operators for the nonperturbatively improved Wilson action, J. High Energy Phys. 08 (2004) 022.

[18] M. Constantinou et al. (ETM Collaboration), Nonperturbative renormalization of quark bilinear operators with $N_{f}=2$ (tmQCD) Wilson fermions and the tree-level improved gauge action, J. High Energy Phys. 08 (2010) 068.

[19] L. H. Karsten and J. Smit, Lattice Fermions: Species doubling, Chiral invariance, and the triangle anomaly, Nucl. Phys. B183, 103 (1981).

[20] M. Bochicchio, L. Maiani, G. Martinelli, G. C. Rossi, and M. Testa, Chiral symmetry on the lattice with Wilson fermions, Nucl. Phys. B262, 331 (1985).

[21] A. T. Lytle and S. R. Sharpe, Nonperturbative renormalization for improved staggered bilinears, Phys. Rev. D 88, 054506 (2013).

[22] A. Bazavov et al. (MILC Collaboration), Scaling studies of QCD with the dynamical HISQ action, Phys. Rev. D 82, 074501 (2010).

[23] A. Bazavov et al. (MILC Collaboration), Lattice QCD ensembles with four flavors of highly improved staggered quarks, Phys. Rev. D 87, 054505 (2013).

[24] A. Hart, G. M. von Hippel, and R. R. Horgan (HPQCD Collaboration), Radiative corrections to the lattice gluon action for HISQ improved staggered quarks and the effect of such corrections on the static potential, Phys. Rev. D 79, 074008 (2009).

[25] S. Borsanyi, S. Durr, Z. Fodor, C. Hoelbling, S. D. Katz et al., High-precision scale setting in lattice QCD, J. High Energy Phys. 09 (2012) 010.

[26] R. J. Dowdall, C. T. H. Davies, G. P. Lepage, and C. McNeile (HPQCD Collaboration), Vus from pi and $\mathrm{K}$ decay constants in full lattice QCD with physical $\mathrm{u}, \mathrm{d}, \mathrm{s}$ and $\mathrm{c}$ quarks, Phys. Rev. D 88, 074504 (2013).

[27] B. Chakraborty, C. T. H. Davies, B. Galloway, P. Knecht, J. Koponen, G. C. Donald, R. J. Dowdall, G. P. Lepage, and C. McNeile (HPQCD Collaboration), High-precision quark masses and QCD coupling from $n_{f}=4$ lattice QCD, Phys. Rev. D 91, 054508 (2015).

[28] D. Guadagnoli, F. Mescia, and S. Simula, Lattice study of semileptonic form-factors with twisted boundary conditions, Phys. Rev. D 73, 114504 (2006).

[29] R. Arthur and P. A. Boyle (RBC and UKQCD Collaborations), Step Scaling with off-shell renormalisation, Phys. Rev. D 83, 114511 (2011).

[30] P. Huber, Renormalization factors of quark bilinears using the DCI operator with dynamical quarks, J. High Energy Phys. 11 (2010) 107.

[31] K. G. Chetyrkin and A. Maier, Wilson expansion of QCD propagators at three loops: Operators of dimension two and three, J. High Energy Phys. 01 (2010) 092.

[32] G. P. Lepage, B. Clark, C. T. H. Davies, K. Hornbostel, P. B. Mackenzie, C. Morningstar, and H. Trottier, Constrained curve fitting, Nucl. Phys. B, Proc. Suppl. 106, 12-20 (2002).

[33] B. Chakraborty, C. T. H. Davies, G. C. Donald, R. J. Dowdall, J. Koponen, G. P. Lepage, and T. Teubner (HPQCD Collaboration), Strange and charm quark contributions to the anomalous magnetic moment of the muon, Phys. Rev. D 89, 114501 (2014). 
[34] S. R. Sharpe and A. Patel, Perturbative corrections for staggered four fermion operators, Nucl. Phys. B417, 307 (1994).

[35] C. T. H. Davies et al. (Fermilab Lattice, LATTICE-HPQCD, and MILC Collaborations), Hadronic-vacuum-polarization contribution to the muon's anomalous magnetic moment from four-flavor lattice QCD, arXiv:1902.04223.

[36] P. Boyle, V. Gülpers, J. Harrison, A. Jüttner, C. Lehner, A. Portelli, and C. T. Sachrajda, Isospin breaking corrections to meson masses and the hadronic vacuum polarization: A comparative study, J. High Energy Phys. 09 (2017) 153.

[37] D. Giusti, V. Lubicz, G. Martinelli, F. Sanfilippo, and S. Simula, Electromagnetic and strong isospin-breaking corrections to the muon $g-2$ from Lattice QCD + QED, Phys. Rev. D 99, 114502 (2019).

[38] I. T. Drummond, A. Hart, R. R. Horgan, and L. C. Storoni, One loop calculation of the renormalized anisotropy for improved anisotropic gluon actions on a lattice, Phys. Rev. D 66, 094509 (2002).
[39] A. Duncan, E. Eichten, and H. Thacker, Electromagnetic Splittings and Light Quark Masses in Lattice QCD, Phys. Rev. Lett. 76, 3894 (1996).

[40] M. Hayakawa and S. Uno, QED in finite volume and finite size scaling effect on electromagnetic properties of hadrons, Prog. Theor. Phys. 120, 413 (2008).

[41] A. Patella, QED corrections to hadronic observables, Proc. Sci., LATTICE2016 (2017) 020 [arXiv:1702.03857].

[42] A. Portelli et al. (Budapest-Marseille-Wuppertal Collaboration), Electromagnetic corrections to light hadron masses, Proc. Sci., LATTICE2010 (2010) 121 [arXiv:1011.4189].

[43] A. Hart, R. R. Horgan, and L. C. Storoni, Perturbation theory versus simulation for tadpole improvement factors in pure gauge theories, Phys. Rev. D 70, 034501 (2004).

[44] Z. Davoudi, J. Harrison, A. Jüttner, A. Portelli, and M. J. Savage, Theoretical aspects of quantum electrodynamics in a finite volume with periodic boundary conditions, Phys. Rev. D 99, 034510 (2019).

[45] G. P. Lepage and P. B. Mackenzie, On the viability of lattice perturbation theory, Phys. Rev. D 48, 2250 (1993). 